\title{
SMHASH: a new mid-infrared RR Lyrae distance determination for the Local Group dwarf spheroidal galaxy Sculptor
}

\author{
Alessia Garofalo, ${ }^{1,2 \star}$ Victoria Scowcroft (ID, ${ }^{3,4 \star}$ Gisella Clementini, ${ }^{1}$ \\ Kathryn V. Johnston, ${ }^{5}$ Judith G. Cohen, ${ }^{6}$ Wendy L. Freedman, ${ }^{7}$ Barry F. Madore, ${ }^{4,7}$ \\ Steven R. Majewski, ${ }^{8}$ Andrew J. Monson, ${ }^{9}$ Jillian R. Neeley, ${ }^{10}$ Carl J. Grillmair, ${ }^{11}$ \\ David Hendel (D), ${ }^{5}$ Nitya Kallivayalil, ${ }^{8}$ Massimo Marengo ${ }^{12}$ and Roeland van der \\ Marel $^{13,14}$ \\ ${ }^{1}$ INAF-Osservatorio di Astrofisica e Scienza dello Spazio di Bologna, via Gobetti 93/3, I-40129 Bologna, Italy \\ ${ }^{2}$ Dipartimento di Fisica e Astronomia-Universitá di Bologna, via Gobetti 93/2, I-40129 Bologna, Italy \\ ${ }^{3}$ Department of Physics, University of Bath, Claverton Down, Bath BA2 7AY, UK \\ ${ }^{4}$ Observatories of the Carnegie Institution of Washington, 813 Santa Barbara St, Pasadena, California, CA 91101, USA \\ ${ }^{5}$ Department of Astronomy, Columbia University, 550 West 120th Street, New York, NY 10027, USA \\ ${ }^{6}$ California Institute of Technology, 1200 E. California Blvd., MC 249-17, Pasadena, CA 91125, USA \\ ${ }^{7}$ Department of Astronomy and Astrophysics, University of Chicago, 5640 S Ellis Ave, Chicago, IL 60637, USA \\ ${ }^{8}$ Department of Astronomy, University of Virginia, Charlottesville, VA 22904-4325, USA \\ ${ }^{9}$ Department of Astronomy and Astrophysics, The Pennsylvania State University, 403 Davey Lab, University Park, PA 16802, USA \\ ${ }^{10}$ Department of Physics, Florida Atlantic University, 777 Glades Rd, Boca Raton, FL 33431, USA \\ ${ }^{11}$ IPAC, Mail Code 314-6, California Institute of Technology, 1200 E. California Blvd., Pasadena, CA 91125, USA \\ ${ }^{12}$ Department of Physics and Astronomy, Iowa State University, Ames, IA 50011, USA \\ ${ }^{13}$ Space Telescope Science Institute, 3700 San Martin Drive, Baltimore, MD 21218, USA \\ ${ }^{14}$ Center for Astrophysical Sciences, Department of Physics and Astronomy, Johns Hopkins University, Baltimore, MD 21218, USA
}

Accepted 2018 August 9. Received 2018 August 9; in original form 2018 May 31

\begin{abstract}
We present a new distance estimation for the Milky Way dwarf spheroidal satellite Sculptor obtained from multi-epoch mid-infrared observations of RR Lyrae stars (RRLs). The $3.6 \mu \mathrm{m}$ observations have been acquired with the Infrared Array Camera onboard the Spitzer Space Telescope as part of the SMHASH program. Mid-infrared light curves for 42 RRLs were obtained, from which we measured Sculptor's distance modulus to be $\mu=19.60 \pm 0.02$ (statistical) \pm 0.04 (photometric) mag (with $\sigma_{\text {sys }}=0.09 \mathrm{mag}$ ), using the $3.6 \mu \mathrm{m}$ empirical period-luminosity relations derived from the Galactic globular cluster M4, or $\mu=19.57 \pm 0.02$ (statistical) \pm 0.04 (photometric) mag (with $\sigma_{\text {sys }}=0.11 \mathrm{mag}$ ) using empirical relations in the same passband recently derived from the Large Magellanic Cloud globular cluster Reticulum. Both these measurements are in good agreement with values presented in previous works with Sculptor RRLs in optical bands, and are also consistent with recent near-infrared RR Lyrae results. Best agreement with the literature is found for the latter modulus which is equivalent to a distance of $d=82 \pm 1$ (statistical) \pm 2 (photometric) kpc (with $\sigma_{\text {sys }}=4 \mathrm{kpc}$ ). Finally, using a subsample of RRLs with spectroscopic metallicities, we demonstrate that these distance estimates are not affected by metallicity effects.
\end{abstract}

Key words: techniques: photometric-stars: distances - stars: variables: RR Lyrae-galaxies: individual (Sculptor) - galaxies: dwarf, Milky Way.

\section{INTRODUCTION}

The processes driving the formation and evolution of dwarf spheroidal (dSph) satellite galaxies around the Milky Way (MW) are still open problems. These systems are precious laboratories, contributing to our understanding of the Universe on both small and large scales - from the formation of the MW stellar halo at the smallest scale, to constraints on cosmological parameters at the largest. The importance of dSphs is based on the assumption that, under the current $\Lambda \mathrm{CDM}$ paradigm, if hierarchical galaxy

^E-mail: alessia.garofalo@unibo.it (AG); v.scowcroft@bath.ac.uk (VS) 
formation theory holds, then these old satellites could be witnesses of the accretion events that led to the formation of the Milky Way's stellar halo (Sales et al. 2007; Stierwalt et al. 2017). The MW satellites are close enough that their stellar populations can be resolved. This offers a unique means to understand the building up of the MW halo by exploiting the information derived from the stars belonging to its satellites.

The different types of pulsating variable stars can help us to distinguish different stellar generations and their radial distributions, particularly when crowding is significant in the host galaxy. It is well known that the dSphs located in the Local Group are characterized by the presence of a significant old stellar component $(t \sim$ 10-13 Gyr) that is dominant in dSphs surrounding the MW (Tolstoy, Hill \& Tosi 2009). RR Lyrae stars (RRLs), besides being the most numerous pulsating variable type, are ever-present, excellent tracers of the old stellar component in $\mathrm{dSphs}$ (for a detailed compilation of the RRLs located in dSphs, see table 3 from Clementini 2010 and table 6 from Martínez-Vázquez et al. 2017). With the distance-gauging precision they afford, these variable stars can also be used to map the 3D structures of nearby galaxies (Clementini 2010; Drake et al. 2013; Belokurov \& Koposov 2016; Gran et al. 2016; Jacyszyn-Dobrzeniecka et al. 2016; Muraveva et al. 2018a, and references therein). The association between dSphs and RRL is so strong that recently these variable stars, as efficient stellar structure indicators, have been used to reveal previously unknown low-luminosity dSphs. Indeed, RRLs are employed both as luminous guides for nearby, faint MW dwarf galaxies $(d<50 \mathrm{kpc}$, $L<1000 \mathrm{~L}_{\odot}$; Sesar et al. 2014) and as lighthouses marking more distant dwarfs $\left(d>50 \mathrm{kpc}, L>1000 \mathrm{~L}_{\odot}\right)$ using current and future deep wide field surveys such as the Large Synoptic Survey Telescope (LSST; Baker \& Willman 2015). Furthermore, RRLs are the primary standard candles for Population II stellar systems widely used to determine distances within our own Galaxy and its nearest neighbours. In fact, thanks to the relation linking the metallicity with the absolute visual magnitude of RRLs $\left(M_{V}(\mathrm{RR})\right.$ $-[\mathrm{Fe} / \mathrm{H}])$, their use as Population II primary distance indicators at optical wavelengths is widespread in nearby galaxies like the MW dSph satellites (see Garofalo et al. 2013, and references therein). After Longmore, Fernley \& Jameson (1986) and Longmore et al. (1990) showed empirically that the RRLs follow a well-defined period-luminosity (PL) relation in the $K$ band, several theoretical and empirical studies came in quick succession (Bono et al. 2001, 2003; Catelan, Pritzl \& Smith 2004; Dall'Ora et al. 2004; Sollima et al. 2006; Coppola et al. 2011; Madore et al. 2013; Braga et al. 2015; Marconi et al. 2015; Muraveva et al. 2015, 2018a,b), to turn the optical-only luminosity-metallicity relation into an extensive, multiwavelength PL relation.

In particular, the Carnegie RR Lyrae Program (CRRP; Freedman et al. 2012) has shown that it is possible with IRAC-Spitzer (Fazio et al. 2004) to measure distances down to 2 per cent accuracy per individual RRL as far as $\sim 60 \mathrm{kpc}$. Moreover, in their recent work on the RRL populations of M4, based on the Galactic calibrator sample (five RRLs with HST trigonometric parallaxes measured by Benedict et al. 2011), Neeley et al. (2017) demonstrated that the dispersion of RRL PL and period-luminosity-metallicity (PLZ) relations decreased to 0.02 mag at Spitzer wavelengths.

To make everything more compelling, the recently occurred Gaia data release 2 (DR2; Gaia Collaboration 2018), next releases to come and their exploitation will provide a powerful help for the scientific community even in this field. Indeed, thanks to Gaia, parallax measurements for hundreds of local RRLs will serve as calibrators to reshape the fundamental relations followed by these stars with unprecedented precision. A taste of Gaia's potential relative to RRL calibration has been shown after the first Gaia data release (Gaia Collaboration 2017), where the parallaxes published are a joint astrometric solution of Tycho and Gaia measurements, Tycho-Gaia Astrometric Solution (TGAS; Lindegren et al. 2016), specific for the first release not adopted for DR2 and next Gaia data releases that all contain Gaia-only astrometry (see e.g. Lindegren et al. 2018, Muraveva et al. 2018c).

The SMHASH program (Spitzer Merger History And Shape of the Galactic Halo; Johnston et al. 2013) moves the study of RRLs in the MW dSphs to mid-infrared (mid-IR) bands where the RRL treatment is advantageously compared with optical bands for the following reasons: (i) the RRL PL intrinsic dispersion at mid-IR bands is narrower, (ii) the RRL light curves are generally more symmetrical and their amplitudes smaller, hence the measurement of the mean magnitudes is more precise, (iii) the extinction effects are weaker and (iv) the mid-IR bands are less dependent on metallicity effects. SMHASH intends to use high-precision mid-IR distances from RRL in the MW halo, its debris streams (e.g. the Sagittarius and Orphan streams; Hendel et al. 2017), and its dSph satellites, to build an accurate three-dimensional map of our Galaxy. These satellites and streams are the residuals of the disruption events that formed the halo; they can be considered 'fossils' of the halo formation. Because they are systems at different stages of dynamical evolution (i.e. different disruption levels), they are the ideal tools to study the processes that occur during the hierarchical build-up of the dark matter (DM) haloes (Bullock, Kravtsov \& Weinberg 2001; Bullock \& Johnston 2005).

SMHASH targeted four MW dSphs: Ursa Minor, Bootes, Sculptor, and Carina. Multiple studies of these four dSphs and their variable star populations have revealed a diversity in their morphological properties. The consistent study of these dSphs - containing from a few tens of RRLs in Bootes (Dall'Ora et al. 2006; Siegel 2006), which also has a distinctly elongated structure, to over 500 RRLs in Sculptor (Martínez-Vázquez et al. 2015) - using SpitzerIRAC will allow us to more tightly constrain their evolutionary history.

Sculptor was the first MW dSph satellite discovered (Shapley 1938) and, consequently, is among the best studied ones. van Agt (1978) identified 602 variables associated with the galaxy, deriving and publishing periods for 64 of these. Kaluzny et al. (1995, hereafter K95), as a part of the Optical Gravitational Lensing Experiment (OGLE) project, have published a catalogue of 229 variables (226 RRLs and three Anomalous Cepheids) located in the inner part of Sculptor. Using the period distribution of RRab stars, these authors estimated that the bulk of Sculptor RRL has a metallicity $[\mathrm{Fe} / \mathrm{H}]_{\mathrm{ZW}} \leq-1.7$ dex. ${ }^{1}$ However, they also note that the colour range spanned by RGB stars is suggestive of a metallicity spread as large as $-2.2 \lesssim[\mathrm{Fe} / \mathrm{H}]_{\mathrm{ZW}} \lesssim-1.6 \mathrm{dex}$. Indeed, Majewski et al. (1999), from an analysis of RGB and HB stars based on optical photometry, found that Sculptor has a bimodal metallicity distribution

\footnotetext{
${ }^{1}$ ZW denotes Zinn \& West (1984) metallicity scale. This scale is widely used in the literature and is based on the average of integrated-light and spectroscopic indices calibrated on a small number of photographic high-resolution spectra. However, different metallicity scales were later developed based on abundance analysis of high-resolution spectra of red giant branch stars in MW globular clusters. A widely used one is the Carretta \& Gratton (1997) scale, which is now superseded by the Carretta et al. (2009) metallicity scale. On average ZW and Carretta et al. (2009) scales differ by only 0.01 dex. Detailed transformation relations between the two scales are provided by Carretta et al. (2009).
} 
with a metal-poor stellar component having $[\mathrm{Fe} / \mathrm{H}] \sim-2.3$ dex and a more metal-rich component at $[\mathrm{Fe} / \mathrm{H}] \sim-1.5$ dex. Combining photometric and high-resolution spectroscopic data, Tolstoy et al. (2004) confirmed the presence of two stellar populations in Sculptor, one metal-rich, $-0.9<[\mathrm{Fe} / \mathrm{H}]<-1.7 \mathrm{dex}$, and one metal-poor, $-1.7<[\mathrm{Fe} / \mathrm{H}]<-2.8 \mathrm{dex}$, that are kinematically and spatially separate from each other. Independently, Battaglia et al. (2008, using the velocity dispersion gradient from the calcium triplet lines in spectra of the galaxy's red giant stars) and de Boer et al. (2011, by measuring the age gradient from the outer to inner galaxy regions with wide-field photometry) confirmed the existence of multiple components in this dSph.

Clementini et al. (2005, hereafter C05) obtained low-resolution spectra for 107 RRLs in Sculptor (about half the sample of RRLs in K95) and measured individual metallicities in the range $-0.85<[\mathrm{Fe} / \mathrm{H}]<-2.40$ dex, with an average value of $[\mathrm{Fe} / \mathrm{H}]_{\mathrm{ZW}}=$ $-1.83 \pm 0.03 \mathrm{dex}(\mathrm{rms}=0.26)$. The study of $\mathrm{C} 05$ remains so far the only spectroscopic measurement study of Sculptor RRL metal abundances. It confirms the existence of a real metallicity spread in this dSph, wider than that found by K95 and consistent with the spread obtained by Geisler et al. (2005) based on high-resolution spectra of four RGB stars $\left(-2.1 \lesssim[\mathrm{Fe} / \mathrm{H}]_{\mathrm{ZW}} \lesssim-0.97 \mathrm{dex}\right)$.

The distance modulus of Sculptor has been measured using several different distance indicators and independent techniques. Over 30 measurements exist in the literature, the majority using RRLs. K95 derived a distance modulus of $19.71 \mathrm{mag}$ based on the average $V$ magnitude of more than 100 RRab stars in their catalogue. Recently, Martínez-Vázquez et al. (2015, 2016a,b) used archival data spanning $24 \mathrm{yr}$ to redouble the known RRL population in Sculptor. They discovered more than 300 new variables spread over $\sim 2 \mathrm{deg}^{2}$ around the galaxy's centre. They used the RRL PL relation in the $I$ band to pin the Sculptor distance modulus down to $19.62 \pm 0.04$ mag. $^{2}$ A detailed comparison of values in the literature for the distance to Sculptor is presented in Section 4.1.

This paper is the first in a series dedicated to the results obtained for the four dSph galaxies observed in the SMHASH program. As Sculptor is the dSph with the largest number of previously known RRLs in our sample, we have chosen this galaxy to demonstrate the observational and data reduction methodologies adopted throughout the dwarf satellites section component of this program. This paper serves as a fiducial work for the rest of the SMHASH program on dSphs.

The paper is organized as follows: observations, data reduction, and the IRAC-Spitzer photometry calibration are presented in Section 2. Section 3 describes the analysis of the RR Lyrae mid-IR light curves and presents the catalogue (atlas). The determination of the distance to Sculptor derived from the RRLs is presented in Section 4 along with a discussion of potential metallicity effects and a comparison with previous distance determinations in the literature. Finally, Section 5 summarizes the paper's main results and conclusions.

\section{OBSERVATIONS AND DATA REDUCTION}

The mid-IR times-series photometry presented in this paper was collected on 2014 October 1-10 with the Infrared Array Camera

\footnotetext{
${ }^{2}$ These authors also inferred (semitheoretical) metallicities for their RRL sample, exploiting the dependence of the $I$-band PL relation on metallicity (Marconi et al. 2015). Their average metallicity is consistent with the majority of previous spectroscopic measurements.
}

(IRAC) on the Spitzer Space Telescope as part of the Warm Spitzer Cycle 10 (PI:61327 K. Johnston). During the Warm Spitzer mission, IRAC operates only using two channels simultaneously, at 3.6 and $4.5 \mu \mathrm{m}$, acquiring observations (12, each with an exposure time of $140 \mathrm{~s}$ ) in two adjacent fields. The SMHASH observations of Sculptor were designed to optimize the signal-to-noise ratio for time-series observations of RRLs in the $3.6 \mu \mathrm{m}$ channel. As a result, the signal-to-noise ratio of individual $4.5 \mu \mathrm{m}$ observations is close to the detection limit. Hence, in this paper we present only the $3.6 \mu \mathrm{m}$ observations.

Our pointing [RA $(J 2000)=01^{\mathrm{h}} 00^{\mathrm{m}} 02.6^{\mathrm{s}}$, DEC $(J 2000)=$ $-33^{\circ} 44^{\prime} 55^{\prime \prime}$ ], about 2 arcsec South-West direction from the galaxy's centre as published in McConnachie (2012), was selected to maximize the number of RRLs in the IRAC field of view, using the RRL catalogues from K95 and C05 as an input. According to the K95 variable star catalogue, there should be 52 RRLs in the 5 arcmin $\times 5$ arcmin region that our pointing covers. In the process of this work, we have found that three of these 52 stars (V1926, V2558, and V2559) have identical coordinates to other stars with different ID numbers, and are undoubtedly the same variables (see Table 1 for details). These doubles had also been noted by MartínezVázquez et al. (2015, 2016b). Therefore, we are left with 49 RRL stars (36 fundamental mode, RRab, and 13 first overtone pulsators, $\mathrm{RRc}$ ) that were observed for 12 epochs, non-uniformly spaced over 10 d. Fig. 1 shows the 49 Sculptor RRLs overplotted on to the Spitzer-IRAC field of view. Blue open circles denote the RRab stars and red filled diamonds denote RRc stars.

Mosaics were created from the Spitzer Science Center S19.2 pipeline processed data and the MOPEX software package (Makovoz \& Khan 2005). A single mosaic was created for each epoch using the dithered exposures. Additionally, a master mosaic using all exposures for all epochs was made as input for the deep ALLFRAME master detection list. Mosaicked location-correction images corresponding to each epoch were also created. PSF photometry was then performed using the DAOPHOT-ALLSTARALLFRAME packages (Stetson 1987, 1994). First, the single-epoch mosaics were converted from MJy/sr to counts (DN) using the conversion factor (MJy/sr per DN/sec) and the exposure times provided in the data headers. Then we ran DAOPHOT, passing through a sequence of routines to obtain a point-spread function (PSF) suitable for all stars of each single-epoch mosaic. Using ALLSTAR, we simultaneously applied the PSF to all stars measured on each single-epoch mosaic. ${ }^{3}$ The average PSF is built selecting from 50 to 90 isolated and bright stars $\left(m_{3.6}<15.5 \mathrm{mag}\right)$ uniformly distributed across the whole frame for each single-epoch mosaic. We then ran ALLFRAME to obtain the final photometry catalogue of sources. ALLFRAME was used to improve the instrumental magnitudes and decrease the photometric uncertainties by fixing the positions of the stars according to the deep reference image and derived translation and rotation of the individual frames, thus reducing the number of free parameters in each PSF fit. The IRAC photometry obtained in this catalogue was calibrated on a zero-point magnitude (zmag) provided in the IRAC handbook ${ }^{4}$ in the standard IRAC Vega magnitude system. The Spitzer photometric calibration is realized by

\footnotetext{
${ }^{3}$ Mosaics have a pixel scale of 0.6 arcsec/pixel, where the typical PSF FWHM value for the Spitzer-IRAC images at $3.6 \mu \mathrm{m}$ is $1.66 \mathrm{arcsec}$, as provided in Section 5 of the IRAC Instrument Handbook, https://irsa.ipac. caltech.edu/data/SPITZER/docs/irac/iracinstrumenthandbook/5.

${ }^{4}$ http://irsa.ipac.caltech.edu/data/SPITZER/docs/irac/iracinstrumenthandb ook (Chapter 4).
} 
Table 1. Properties of RRLs in Sculptor observed at $3.6 \mu \mathrm{m}$. The variables are ordered by increasing ID number, adopting the nomenclature from K95 and C05. Column 1 lists the star identifier; columns 2 and 3 provide the right ascension and declination (J2000 epoch), respectively. The coordinates were obtained from our astrometrized reference image (see Section 2). Column 4 lists the classification of RRL according to pulsation mode, fundamental-mode (RRab), or first-overtone (RRc) pulsators; columns 5, 6, and 7 list the pulsation period, its logarithm, and $\mathrm{HJD}_{\max }$ from either K95 or C05 (if a spectroscopic metallicity is available in column 13, then the values in columns 5, 7, 8, and 9 come from C05, otherwise, they are from K95). Columns 8 and 9 list, respectively, the intensity-weighted mean magnitudes and amplitudes of the light variation in the $V$ band from either $\mathrm{K} 95$ or $\mathrm{C} 05$, while columns 10,11 , and 12 give the intensity-weighted mean magnitudes, the corresponding errors, and the amplitudes of the light variation at $3.6 \mu \mathrm{m}$. Finally, column 13 provides spectroscopic metallicities from $\mathrm{C} 05$, where available.

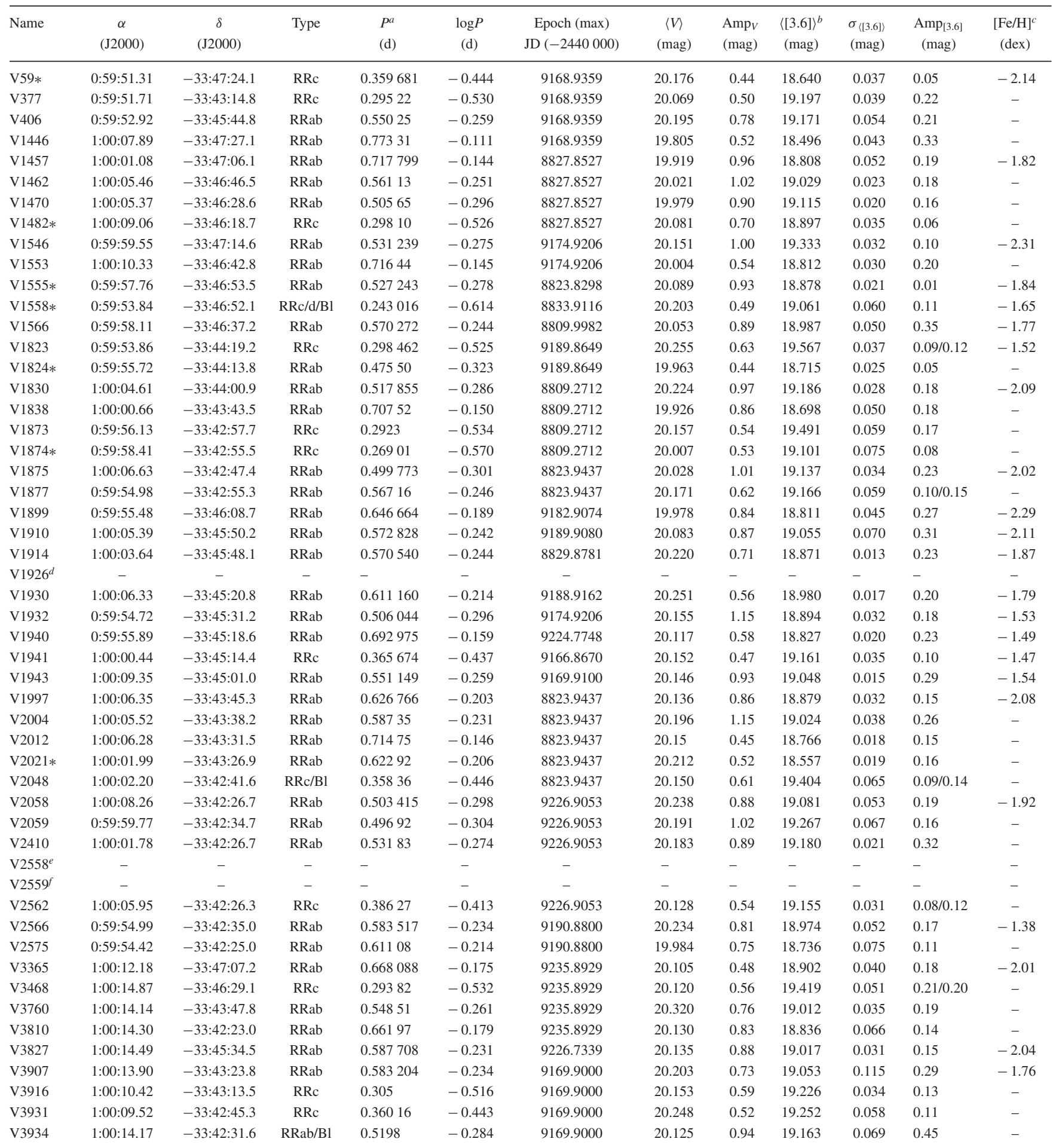

Notes. ${ }^{a}$ Period, epoch of maximum light, and intensity-averaged mean $V$ magnitude for stars with metallicities are from $\mathrm{C} 05$, who determinated them anew from the light curves based on data from K95. ${ }^{b}$ The [3.6] mean magnitudes presented in this work are not reddening corrected. Assuming a foreground $E(B-V)=0.0159$ mag from the Schlafly \& Finkbeiner (2011) map and applying the Monson et al. (2012) equation for $3.6 \mu \mathrm{m}\left[A_{3.6}=0.203 \times E(B-V)\right]$, the extinction measured for Sculptor is one order of magnitude smaller than the photometric error (i.e. $A_{3.6} \leq 0.003 \mathrm{mag}$ ) and can be safely neglected for the purposes of this study. ${ }^{c}$ Spectroscopic metallicities from C05 on the Zinn \& West scale. ${ }^{d}$ Same coordinates as V406. ${ }^{e}$ Same coordinates as V2058. ${ }^{f}$ Same coordinates as V2059. $*$ Most problematic stars. 


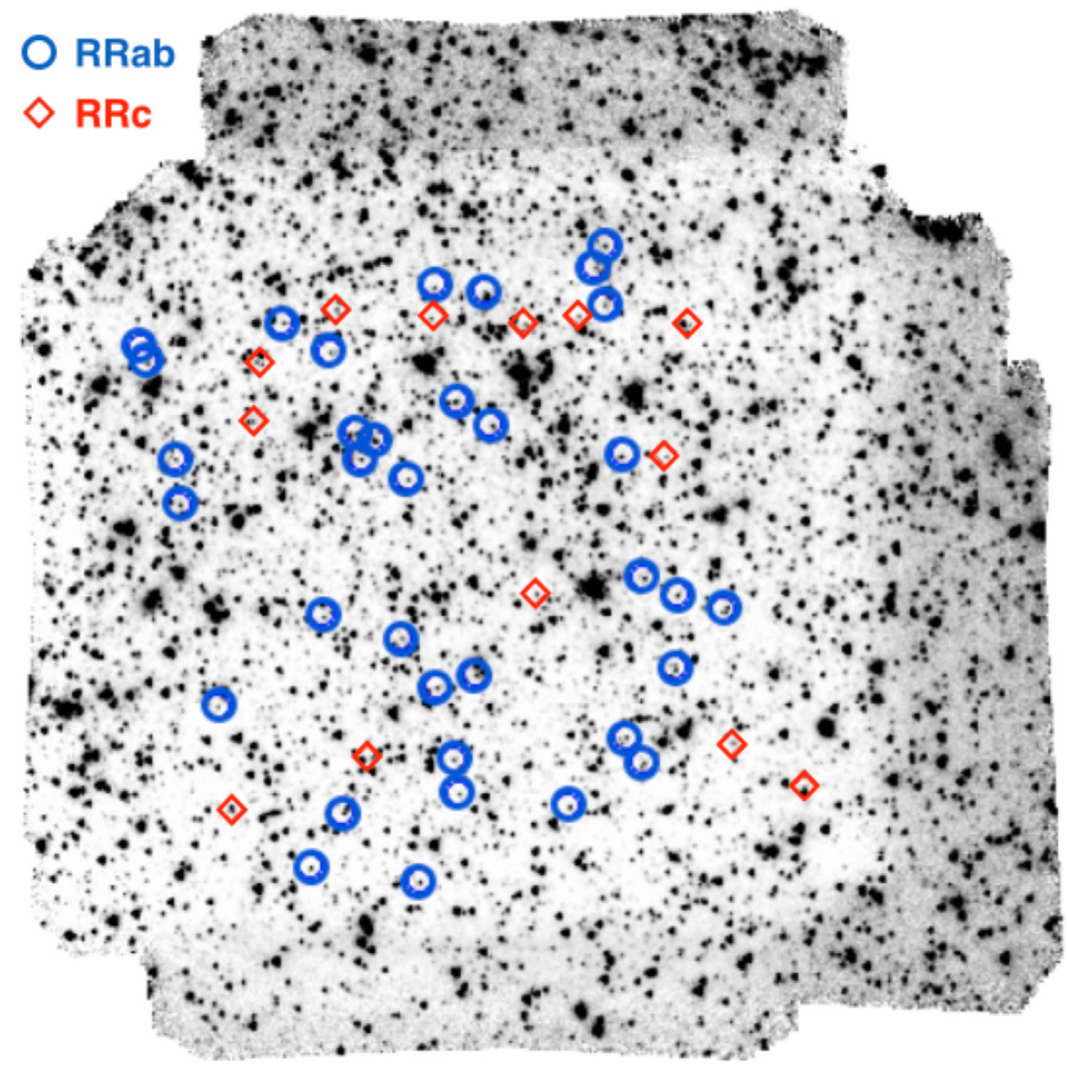

Figure 1. IRAC-Spitzer master mosaic at $3.6 \mu \mathrm{m}$ that shows the 49 Sculptor RRLs in our sample encircled by blue open circles (RRab stars) or red diamonds (RRc stars). The field of view of the IRAC frame is $5 \operatorname{arcmin} \times 5$ arcmin. North is up and east is to the left.

applying an aperture correction to the list of sources chosen to measure the PSF. This step is implemented using DAOPHOT routines in the $\mathrm{IRAF}^{5}$ environment. A final PSF correction (or calibration) is then calculated from the PSF photometry and the corrected aperture photometry, which is then applied to all stars. This transforms all the IRAC photometry on to the IRAC Vega system. The final step is the application of the array location-dependent correction on to the photometric measurements of the final catalogue using a set of correction images. The corrections depend on the exact position of sources on these images created concurrently with the single-epoch mosaics (detailed information on this process and the creation of the correction images are explained in the IRAC handbook ${ }^{6}$ ).

Seven out of the 49 RRLs on our frames are very close to other objects, or else have an elongated shape, which makes it difficult to measure their PSF profile accurately, thus making their photometry unreliable. These stars are marked with asterisks in Table 1. Such effects are visible in the light curves of these stars (see Section 3) and have repercussions for their positions on the [3.6]- $\log P$ plane (see Section 4). We have discarded these seven stars (V59, V1482, V1555, V1558, V1824, V1874, V2021) from our PL study. Accordingly, we start our analysis with a sample of 42 RRLs.

${ }^{5}$ IRAF is distributed by the National Optical Astronomical Observatory, which is operated by the Association of Universities for Research in Astronomy, Inc., under cooperative agreement with the National Science Foundation.

${ }^{6}$ http://irsa.ipac.caltech.edu/data/SPITZER/docs/irac/iracinstrumenthandb ook (Section 4.5).

\section{LIGHT CURVES}

The light curves of the variables were analysed with the Graphical Analyzer of Time Series (GRATIS) software, which was developed at the Bologna Observatory by P. Montegriffo (see e.g. Clementini et al. 2000). Our data were not optimized for period searches, thus we built the light curves using reference periods and times of maximum light $\left(\mathrm{HJD}_{\max }\right)$ from $\mathrm{K} 95$ and $\mathrm{C} 05$.

All 42 of our variables have been studied by K95 but only 23 are in common with $\mathrm{C} 05$, who measured spectroscopic metallicities and remeasured the periods and $\mathrm{HJD}_{\max }$ from the $\mathrm{K} 95$ data. Where available, we use the periods and $\mathrm{HJD}_{\max }$ from $\mathrm{C} 05$, otherwise we use the original values from K95. The K95 periods and those redetermined by $\mathrm{C} 05$ differ only at the 4 th or 5 th decimal digit onwards. We consider these changes negligible. For one star, V1875, C05's period differs significantly from that derived by K95. C05 classified V1875 as an RRab while K95 considered it an RRc. We found that the C05 period and classification for V1875 fit our data well (see Fig. 2), and so we retain the C05 period (and hence classification) for consistency within our analysis. The final sample in our catalogue is as follows: 33 fundamental-mode (RRab) and 9 first-overtone pulsators (RRc). The light curves of the variable stars are presented in Fig. 2 while the time-series data of each RRL at $3.6 \mu \mathrm{m}$ are provided in Table 2 , which is published in its entirety in the electronic version of the journal.

While identifying and classifying the variables, K95 found that some are possibly affected by the Blažko effect (a cyclical effect that causes modulations in periods and amplitudes of the light curves; Blažko 1907). Three of these stars are in our sample: V1558, V2048, 
[3.6]

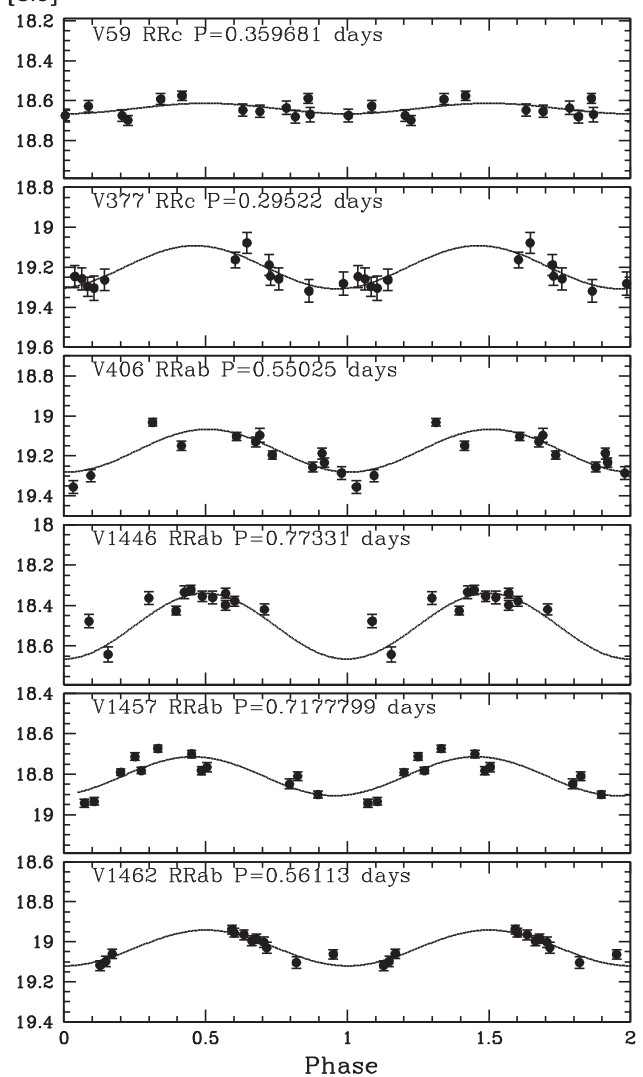

[3.6]

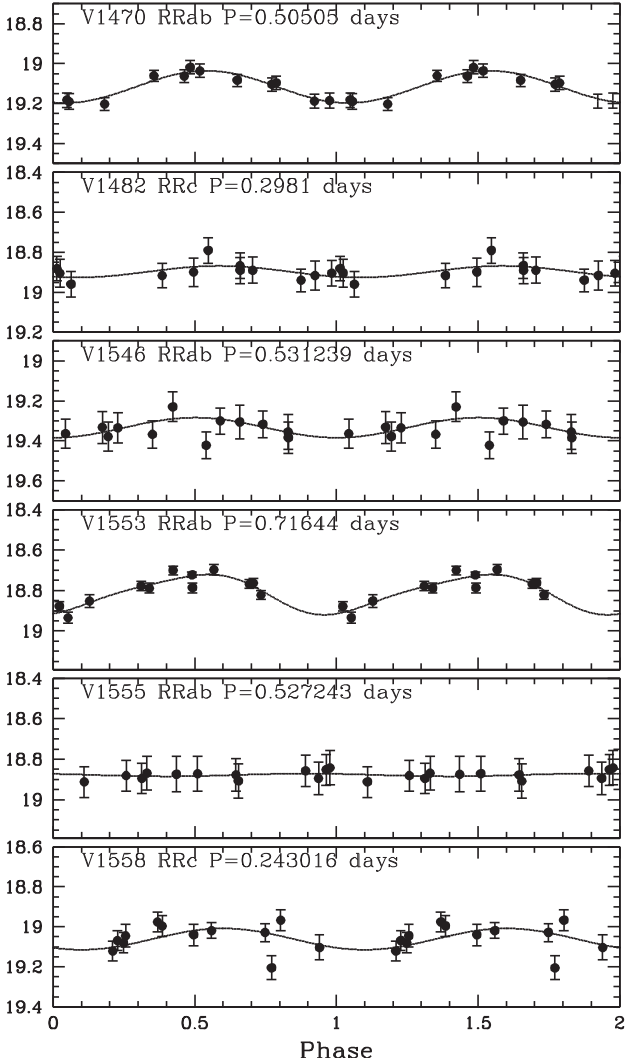

Figure 2. Light curves in the [3.6]-band for our sample of 49 RRLs in the Sculptor galaxy. The stars are ordered with increasing ID number adopting the nomenclature from K95 and C05. The solid black lines represent the light-curve models generated by GRATIS using all phase points. V59, V1482, V1555, and V1558 are problematic stars not used to fit the PL relations (see Section 3.1 and Table 1). The first 12 light curves are shown here, and the remaining are plotted as supplementary information available in the online journal.

Table 2. Photometry of Sculptor RRL at $3.6 \mu \mathrm{m}$.

\begin{tabular}{lcc}
\hline $\begin{array}{l}\text { HJD } \\
(-2450000)\end{array}$ & $\begin{array}{r}\text { V377 }- \text { RRc } \\
{[3.6]} \\
(\mathrm{mag})\end{array}$ & $\begin{array}{l}\sigma_{[3.6]} \\
(\mathrm{mag})\end{array}$ \\
\hline 6932.465260 & 19.246 & 0.055 \\
6933.335707 & 19.281 & 0.058 \\
6933.848714 & 19.188 & 0.053 \\
6934.994379 & 19.163 & 0.040 \\
6935.732669 & 19.305 & 0.061 \\
6936.220511 & 19.259 & 0.055 \\
6937.219967 & 19.263 & 0.054 \\
6938.087896 & 19.297 & 0.048 \\
6938.967365 & 19.259 & 0.055 \\
6939.499397 & 19.319 & 0.057 \\
6940.344800 & 19.245 & 0.045 \\
6941.206090 & 19.078 & 0.052
\end{tabular}

Note. The table is available in its entirety in an electronic form in the online journal. A portion is shown here for guidance regarding its form and content.

and V3934. Moreover, we find that V1558 and V2048 are significantly affected by crowding and/or blending. Visual inspection of our imaging also reveals that some of our other stars lie in crowded regions (e.g. V59), or are clearly blended with neighbour stars or diffuse objects. We will discuss these problematical objects in detail in the following subsection.

\subsection{Comments on individual problematic stars}

The following stars are discussed from the most to the least problematic source. The first seven stars (V59, V1482, V1555, V1558, V1824, V1874, V2021), which could be clearly identified as contaminated from the initial visual inspection of the images, are not included in any PL analysis. This is clearer in Fig. 3, which shows the mid-IR period-amplitude diagram of the 42 RRLs together with the seven discarded sources, based on light curves at $3.6 \mu \mathrm{m}$ : most of the RRLs excluded (black crosses) have mid-IR amplitude smaller than the RRab (blue open circles) and RRc stars (red diamonds) of the sample. We include the details here for completeness:

V59 - Kovács (2001) classifies star V59 as a suspected doublemode (RRd) with periods of $0.35968 / 0.4837 \mathrm{~d}$. C05 do not confirm this finding, and instead suggest a classification of a monoperiodic RRc with a noisy light curve. A visual inspection of our images shows it to be completely blended with a nearby star. This is reflected in the time-series as an unnaturally flattened lightcurve.

V1482 - The star is the second brightest RRc in our sample. The light curve's amplitude is smaller than expected (see Fig. 3). It is possibly a blended source. The presumed blending is further confirmed by visual inspection of WFC3@HST images (PI:12966 van der Marel), overlapping our IRAC pointing and having higher spatial resolution ( $0.04 \mathrm{arcsec} / \mathrm{pixel})$.

V1555 - This star is very close to two sources, one of which is brighter than V1555. The bright source may be contaminating the 


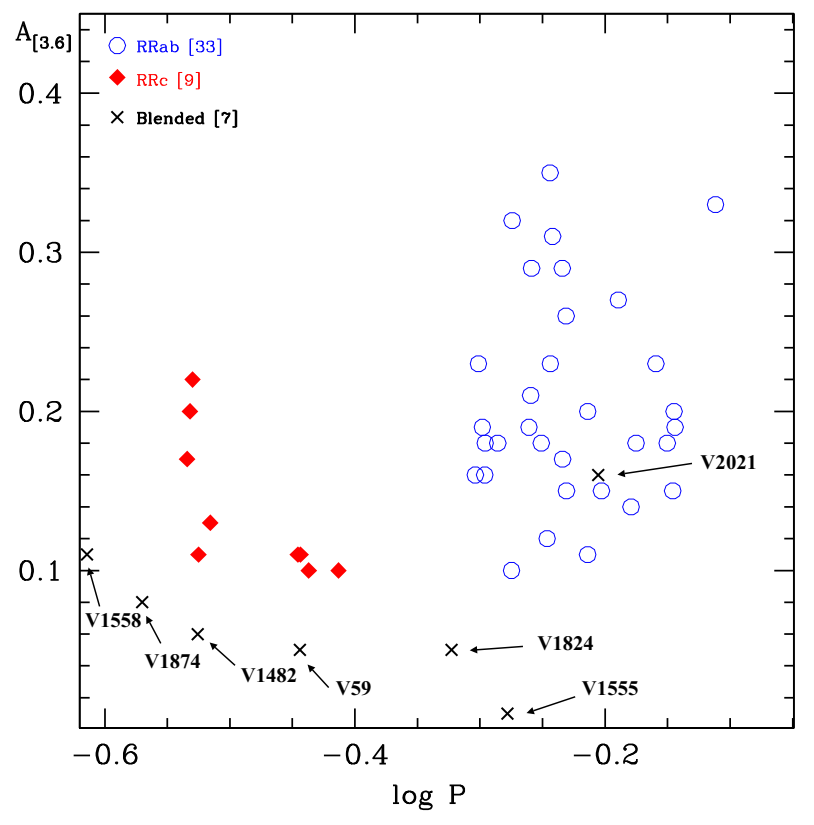

Figure 3. Mid-IR period-amplitude diagram for all the RRLs in our sample based on light curves at $3.6 \mu \mathrm{m}$. The blue open circles and the red diamonds mark the RRab and RRc stars, respectively. The RRLs affected by crowding/blending and discarded in this study are labelled and marked with crosses.

flux measurement of V1555, polluting the light-curve periodicity and the mean magnitude (see Figs 2-4).

V1558 - Suspected Blažko in K95, whereas C05 suspected it may be an RRd star. In our images V1558 has an elongated shape, which is probably due to the contamination from a background source.

V1823 - This is the faintest star of our sample but falls within $1 \sigma$ of our PL relations and also fits well to the PL relations of Neeley et al. (2015) and Madore et al. (2013). One data point was removed from the light-curve fit (grey filled squares in Fig. A1) as it significantly deviated from the fitted light-curve model, but is shown in the figure for completeness.

V1824 - Visual inspections of the light curve, images, and log $P$-luminosity plane show that the star is clearly blended with a close diffuse source. The light-curve amplitude is reduced (Figs 3 and A1) and the mean magnitude is at least 0.5 mag brighter than expected (Fig. 4).

V1874 - The light curve is noisy, most likely caused by blending from a close neighbour object indistinguishable on the images.

V2021 - There are not any caveats in the literature regarding this object, but in our images it appears rather extended. This is most likely due to crowding/blending and the reduced resolution of the IRAC images compared to previous studies.

V1877 - The light curve is not sampled between $\phi=0.9$ and 1.3 (the non-uniform cadence of our observations means that we would never optimally sample the light curve of every star in the field) and, like V1823, has one data point that has been excluded from the fit. However, its mean magnitude plotted on the [3.6]-log $P$ plane follows the PL relations well.

V2048 - According to K95 possibly a Blažko source. Classified by Kovács (2001) as an RRd star.

V2562 - The light curve shows one data point that has been excluded from the fit but this does not affect the star's mean magnitude.
V3931 - K95 considered this star to be an RRc with a period of 0.36016 d. Kovács (2001) classifies this star as an RRd star with periods $0.358350 / 0.48140 \mathrm{~d}$.

V3468 - Adopting the period and $\mathrm{HJD}_{\max }$ from K95, the light curve is not fully sampled and a couple of data points are significant outliers. Although our data were not optimized for period searches, we attempted to perform a search using GRATIS. GRATIS uses a Lomb periodogram to search for candidate periods within the typical RR Lyrae interval, then uses the Fourier truncated series to complete the period definition by finding the best-fitting model using the additional information the light curve provides - i.e. the amplitude, average luminosity of the variable, and the root mean square (rms) between the data points and the best-fitting model. The period found using GRATIS $(P=0.2733 \mathrm{~d})$ was shorter than the one found by K95 ( $P=0.2938 \mathrm{~d})$, and gives a light-curve fit with both smaller rms ( 0.04 from the GRATIS period compared to 0.06 using the K95 period) and smaller $\chi^{2}$ (see Fig. A3). However, because we did not design our observations with a view to perform period searches, systematics may be present that could affect this measurement. An analysis of such uncertainties is beyond the scope of this work, so rather than trust the new measurement unequivocally we test both values in our analysis.

V1875 - The classification of both type and period differs significantly between K95 and C05. The formers consider the star to be an RRc affected by the Blažko effect, while C05 considered the star to be an RRab. The periods published by the two studies are aliased $\left(P_{\mathrm{K} 95}=0.66 P_{\mathrm{C} 05}\right)$. Our observations are in agreement with the $\mathrm{C} 05$ findings.

V3934 - K95 consider this a possible Blažko source. We do confirm their period.

The Blažko effect appears generally as a variation of amplitude and period of the light curve typically occurring on time-scales from a few tens to a few hundreds of days. This effect has not yet been investigated in detail at mid-IR wavelengths but it is likely that the amplitude modulation is reduced when going from shorter to longer wavelengths (Gavrilchenko et al. 2014; Klein et al. 2014). Furthermore, the time interval covered by our observations may be too short to reveal the Blažko modulation. These may be the reasons why the amplitude and mean magnitude values we obtain are normal for an RRL with this period.

\section{PERIOD-LUMINOSITY RELATIONS AND DISTANCE DETERMINATIONS}

Mid-IR PL relations for RRLs have appeared rather recently. Madore et al. (2013) derived PL relations for the Wide-field Infrared Survey Explorer (WISE) mid-IR bands at 3.4, 4.6, and $12 \mu \mathrm{m}$. They adopted from the Galactic field four RRab stars (brighter than $V \sim 9$ mag) with trigonometric parallaxes measured by Benedict et al. (2011) using the Fine-Guidance Sensor cameras on the $\mathrm{Hub}$ ble Space Telescope (HST; FGS@HST) as calibrators. Since then, Dambis, Rastorguev \& Zabolotskikh (2014) have published WISE PL relations using RRL ( $\sim 360$ stars up to $V \sim 15 \mathrm{mag}$ ) belonging to 15 Galactic globular clusters at distances of not more than 15 kpc. Almost concurrently, Klein et al. (2014) presented RRL PL relations using the AllWISE Data Release, adopting a sample of 129 RRLs with distances up to $2.5 \mathrm{kpc}$ (brighter than $V \sim 13 \mathrm{mag}$ ). Both of these latter studies, as done by Madore et al. (2013), derived their relations by tying their zero point to the four Galactic RRab stars for which HST parallax values are available. 


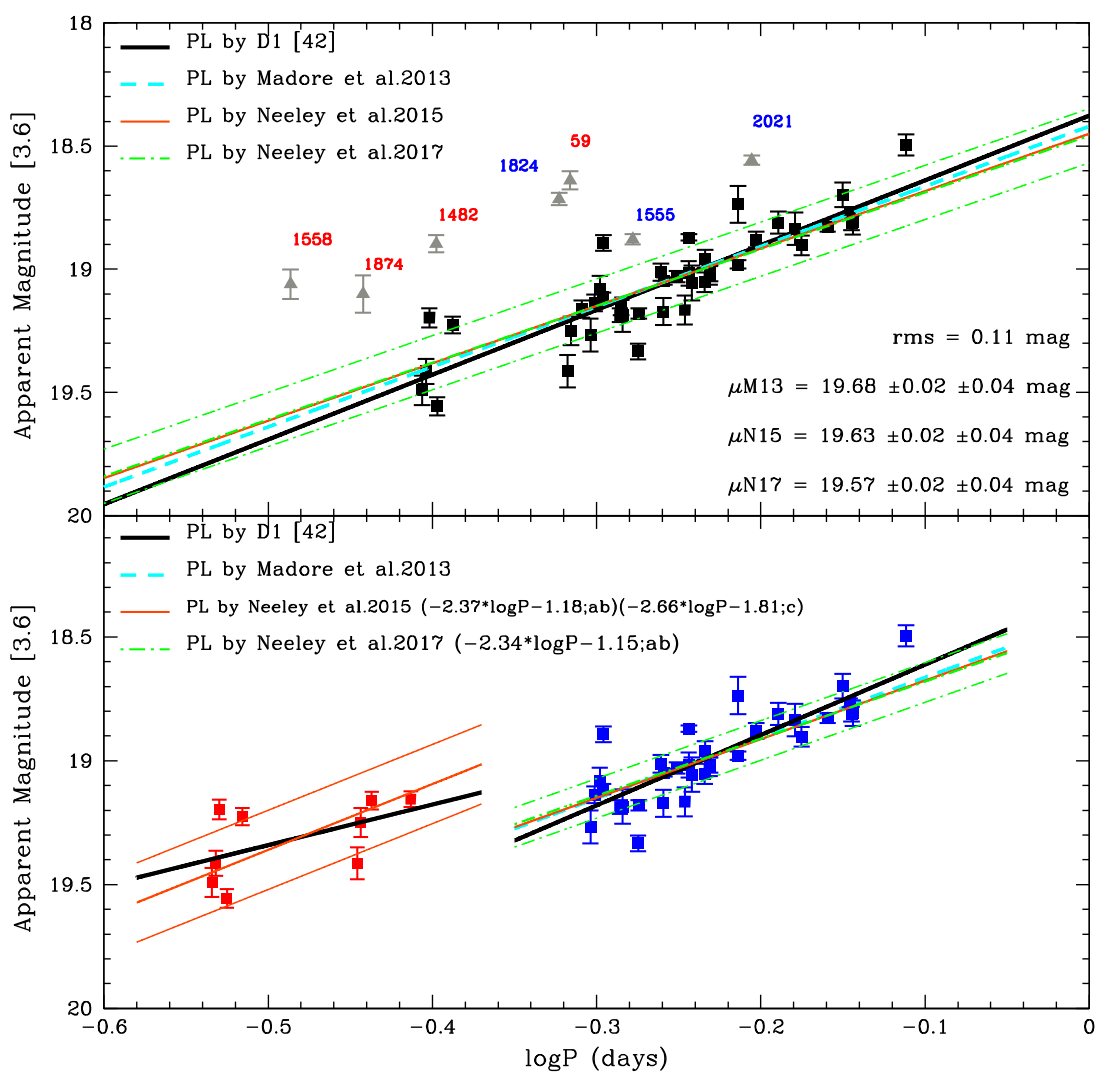

Figure 4. Top: $3.6 \mu \mathrm{m}$ PL relations defined by the 42 RRLs observed in Sculptor (D1 sample). The periods of the RRc stars have been fundamentalized. The black solid line represents the least-squares fit to the data in Table 1. The triangles are the most problematic stars not used in the least-square fit. Bottom: RRab (blue symbols) and RRc (red symbols) stars are plotted in two PL relations that were computed from the fundamental-mode and the first-overtone pulsators separately. In both panels, the cyan (dashed), orange (thin solid), and green (dash-dot) lines mark Madore et al. (2013), Neeley et al. (2015), and Neeley et al. (2017) PL relations, respectively. We show only $\pm 2 \sigma$ the deviation lines of the Neeley et al. (2017)'s PL; the dispersions of the other PL relations are similar or slightly broader.

Neeley et al. (2015) measured PL relations at 3.6 and $4.5 \mu \mathrm{m}$ using the five Galactic RRLs with trigonometric parallaxes from HST [Benedict et al. 2011; four RRab stars used by Madore et al. (2013), Dambis et al. (2014), and Klein et al. (2014), plus 1 RRc] as zeropoint calibrators and IRAC-Spitzer observations of 37 RRLs in the globular cluster M4. They provided PL relations both for the combined RRab+RRc sample and the separate RRab and RRc samples (table 3 of Neeley et al. 2015). Neeley et al. (2017) also derived theoretical PLZ relations in the mid-IR using non-linear, timedependent convective hydrodynamical models. Comparing these theoretical PLZ relations with a sample of RRLs from the CRRP with IRAC photometry and spectroscopic metallicities from Monson et al. (2017), they showed that the mid-IR PLZ can provide distances to individual RRL with uncertainties better than 2 percent. The limiting factor in the systematic accuracy of mid-IR PLZs is the small sample of RRL with precise enough parallax values that can be used as calibrators. Most recently, Muraveva et al. (2018b) derived PL relations at the 3.6 and $4.5 \mu \mathrm{m}$ Spitzer passbands from a sample of 24 RRLs in the Large Magellanic Cloud (LMC) globular cluster Reticulum. The zero points of their PL relations were estimated using Benedict et al. (2011) HST parallaxes for the five Galactic RRL calibrators and, as an alternative, the trigonometric parallaxes published in Gaia Data Release 2 (DR2) for four of the same stars (Lindegren et al. 2018). The Gaia DR2 parallax of the fifth star (RR Lyr itself, the prototype of the whole class) has a wrong value (Arenou et al. 2018, Gaia Collaboration 2018), therefore for
RR Lyr Muraveva et al. (2018b) used Gaia DR1 parallax that was obtained as part of the TGAS. Muraveva et al. (2018b) find that the two calibration procedures provide consistent results within the respective errors. This will improve dramatically with subsequent data releases from Gaia, when the sample of Galactic RRLs that have the required high-precision parallax determinations, mid-IR photometry, and spectroscopic metallicities, that can be used as calibrators is expected to increase by at least an order of magnitude.

In this work, we derived our PL relations using the starting sample of 42 Sculptor RRLs that we divided in various different subsamples as summarized in Table 3.

The PL relations are presented in Figs 4 and 5 for two of these subsamples. In the top panel of each figure, the periods of the RRc stars have been fundamentalized, i.e. the first overtone pulsators were transformed into fundamental pulsators modifying their periods according to the equation

$\log P_{f}=\log P_{R R c}+0.127$

(Iben 1974) and the RRab and RRc have been fitted together using a single PL relation. In the bottom panels, we plot the RRab and RRc separately and fit them using independent PLs. The grey filled triangles mark the seven most problematic variables (i.e. V59, V1482, V1555, V1558, V1824, V1874, V2021), whose images and light curves reveal signatures of blending and crowding (in some cases confirmed by $\mathrm{K} 95$ and $\mathrm{C} 05$ ). The thick black solid line is the least-squares fit calculated by excluding the seven blended stars. 
Table 3. Definition of data sets used in this analysis.

\begin{tabular}{|c|c|c|}
\hline $\begin{array}{l}\text { Sample } \\
\text { identifier }\end{array}$ & Description & $\begin{array}{l}\text { No. RRL } \\
(\text { RRab+RRc) }\end{array}$ \\
\hline D1 & Full sample. Original light curves. & $42(33+9)$ \\
\hline D2 & $\begin{array}{l}\text { Six partially problematic stars removed } \\
\text { (see Section 3.1). }\end{array}$ & $36(32+4)$ \\
\hline $\mathrm{D} 3^{a}$ & $\begin{array}{l}\text { Full sample. Stars removed in D2 have } \\
\text { corrected light curves. V3468 has } \\
\text { GRATIS period. }\end{array}$ & $42(33+9)$ \\
\hline $\mathrm{D} 3^{b}$ & $\begin{array}{l}\text { Full sample. Stars removed in D2 have } \\
\text { corrected light curves. V3468 has K95 } \\
\text { period. }\end{array}$ & $42(33+9)$ \\
\hline D4 & Strict selection based on FourStar images. & $19(17+2)$ \\
\hline $\mathrm{DZ}^{c}$ & $\begin{array}{l}\text { Stars with spectroscopic metallicities } \\
\text { from } \mathrm{C} 05 .\end{array}$ & $20(18+2)$ \\
\hline
\end{tabular}

Notes. ${ }^{a}$ Adopting the GRATIS period for V3468 $(P=0.2733 \mathrm{~d}) .{ }^{b}$ Adopting the K95 period for $\mathrm{V} 3468(P=0.2938 \mathrm{~d}){ }^{c}$ Sample contains $14 \mathrm{RRLs}$ defined as metal-poor (with $[\mathrm{Fe} / \mathrm{H}]<-1.7 \mathrm{dex}$ ), 6 RRLs defined as metal-rich (with $[\mathrm{Fe} / \mathrm{H}]>-1.7 \mathrm{dex})$.

The cyan (dashed), orange (thin solid), and green (dot-dashed) lines use the slopes of the empirical PL relations from Madore et al. (2013), Neeley et al. (2015), and Neeley et al. (2017), respectively. To avoid overcrowding the figures, we only show the $\pm 2 \sigma$ deviation lines of Neeley et al. (2017) as an example; dispersions in the other PLs are similar.

Recall from Section 3 that the seven most problematical stars have already been discarded from the sample and are not considered in any of the following analysis. We refer to our starting sample from this point forward as dataset1 (D1; 42 RRL, $33 \mathrm{RRab}+9$ $\mathrm{RRc}$ ). The scatter from our best-fitting model is $0.11 \mathrm{mag}$, which is smaller than the previous work based on infrared photometry (Pietrzyński et al. 2008; $\sigma_{K}=0.22 \mathrm{mag}, \sigma_{J}=0.21 \mathrm{mag}$ ). These authors suggest that the single-phase nature of their observations and the metallicity spread of the galaxy might contribute to the large scatter they observe. We will investigate the role played by metallicity later in this section.

To determine the quality of our photometry and to see how any systematics arising from blending and/or crowding might affect our results, we subdivided our sample into several data sets (see Table 3). This allows us to choose the most accurate sample of RRL to measure the distance modulus of Sculptor. Dataset2 (D2) contains 36 stars, the 'good' variables that remain if we exclude the six partially problematic stars (V1823, V1877, V2048, V2562, V3931 with one or two phase points excluded in the light-curve best fits and V3468 with an ambiguous period, see Section 3.1). The dataset3 (D3a), which comprises 42 stars: 36 'good' variables and the six partially problematic stars with the average magnitude calculated for five of them by using only the 10 or 11 phase points that best fit the light-curve model. For V3468 the revised period determined in Section 3 using GRATIS and our data is adopted. Instead, dataset3b (D3b) is the same as in D3a except for V3468's period, which is provided by $\mathrm{K} 95$.

Table 4 summarizes the slopes of the PL relations for the RRabonly, RRc-only, or combined RRab+RRc samples, measured by least-square fitting each of the data sets D1, D2, and D3 (columns 2, 3,4 , and 5, respectively). A comparison among data sets shows that the slopes of the RRab+RRc and RRab relations are all compatible within their errors. The RRc stars in our sample exhibit a slope that differs significantly from the slopes of the RRab and RRab+RRc samples and even more so from Neeley et al. (2015)'s slope for
RRc stars (Table 4, column 8). However, the large uncertainties on our RRc slope results mean that the difference is not statistically significant. Neeley et al. (2015)'s slope for RRc stars also differs from and is steeper than that for RRab, but our RRc slopes go in the opposite direction and are shallower. One possible explanation of this finding is the small number and poor distribution in period of the RRc stars in our sample. There are approximately double the number of RRab compared to RRc stars in our sample. However, because RRc stars are fainter and hence more likely to suffer blending by bright neighbours than RRab stars, after discarding problematical stars the RRc in the D1 sample reduced approximately to one third the number of RRab stars (and lower still in the D2 sample). In Fig. 4, the bottom panel, it is clear that the RRc stars do not cover the periods range entirely, but cluster around $\log P \approx-0.425$ and -0.525 . The poor RRc period distribution means that their PL slopes are less robust than the RRab-only and RRab+RRc slopes. The combination of the non-uniform period distribution with the reduced sample size results in significantly higher uncertainties for the RRc-only samples. For this reason, we exclude the RRc-only samples from further analysis in this work.

As a final test on the quality of our sample, we compared our deepest image with a deep ground- based $H$-band image of Sculptor from the FourStar NIR camera on the $6.5 \mathrm{~m}$ Magellan telescope (Persson et al. 2013). The image covers a larger area than the SpitzerIRAC field of view, with a resolution of 0.68 arcsec and an exposure time of $1000 \mathrm{~s}$. A comparison with this higher resolution, deeper image allows us to select only those RRLs that are perfectly isolated in the Spitzer images to measure the distance to Sculptor.

The outcome of this strict selection is our final quality-test subset, D4. It consists of 19 RRLs (17 RRab + 2 RRc). Fig. 5 shows the PL relations for the D4 sample, colours, and symbols are the same as in the previous figures. Column 6 of Table 4 lists the slopes of the PL relations from D4. Since there are only two RRc stars in the D4 sample, we did not attempt to measure an RRc-only slope. There is a perfect agreement between the RRab and RRc+RRab slopes of the PL relations from D4. These slopes are shallower than found from the D1, D2, and D3 data sets and in very good agreement with the slopes found by Muraveva et al. (2018b) from RRLs in the Reticulum cluster. They also agree well within the errors with the slopes in Madore et al. (2013), Neeley et al. (2015), and Neeley et al. (2017).

\subsection{Distance determinations}

Since our main aim is to measure the Sculptor distance modulus from our data, we adopt the following mid-IR PL relations of RRab and RRab+RRc as fiducials:

For RRab+RRc:

$M_{[3.4]}=-2.44( \pm 0.95) \log (P)-1.26( \pm 0.25)$

$\sigma=0.10 \mathrm{mag}$, equation (1) of Madore et al. (2013);

$M_{[3.6]}=-2.332( \pm 0.106) \log (P)-1.176( \pm 0.080)$

$\sigma=0.095 \mathrm{mag}$, equation (4) of Neeley et al. (2015);

$M_{[3.6]}=-2.304( \pm 0.105) \log (P)-1.112( \pm 0.089)$

$\sigma=0.055 \mathrm{mag}$, from Neeley et al. (2017);

$M_{[3.6]}=-2.15( \pm 0.23) \log (P)-1.19( \pm 0.05)$

$\sigma=0.06 \mathrm{mag}$, from Muraveva et al. (2018b) calibrated on the five Galactic RRLs with $H S T$ parallaxes.

$M_{[3.6]}=-2.15( \pm 0.23) \log (P)-1.08( \pm 0.03)$ 


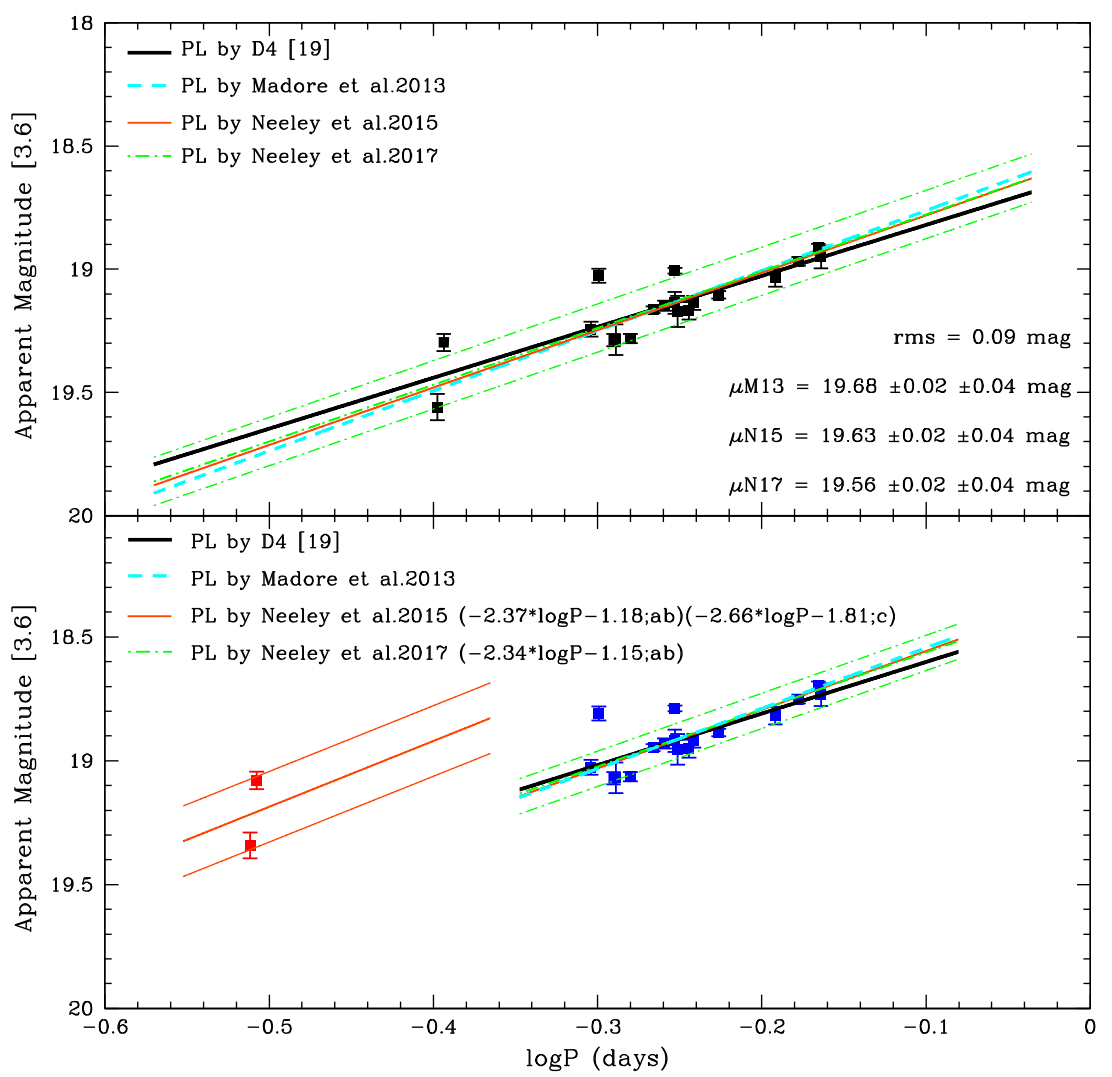

Figure 5. $3.6 \mu \mathrm{m}$ PL relations defined by the 19 RRLs that compose the D4 sample. Symbols, colours, and $\pm 2 \sigma$ dispersion lines are as in Fig. 4.

Table 4. Slopes of the [3.6] PL relations obtained for different selections of Sculptor RRab+RRc, RRab, and RRc stars.

\begin{tabular}{|c|c|c|c|c|c|c|c|c|c|}
\hline Sample & D1 [42] & D2 [36] & $\mathrm{D} 3[42]^{a}$ & D3 $[42]^{b}$ & D4 [19] & $\begin{array}{l}\text { Madore et al. } \\
\text { (2013) }\end{array}$ & $\begin{array}{l}\text { Neeley et al. } \\
\text { (2015) }\end{array}$ & $\begin{array}{l}\text { Neeley et al. } \\
\text { (2017) }\end{array}$ & $\begin{array}{l}\text { Muraveva et al. } \\
\quad(2018 b)\end{array}$ \\
\hline \multicolumn{10}{|l|}{ RRab+RRc } \\
\hline \multicolumn{10}{|l|}{ RRab } \\
\hline slope $\pm \sigma$ & $-2.84 \pm 0.33$ & $-2.82 \pm 0.33$ & $-2.85 \pm 0.33$ & $-2.85 \pm 0.33$ & $-2.08 \pm 0.41$ & $-2.44 \pm 0.95$ & $-2.37 \pm 0.14$ & $-2.342 \pm 0.140$ & - \\
\hline $\mathrm{rms}$ & 0.10 & 0.10 & 0.10 & 0.10 & 0.08 & 0.10 & - & 0.040 & - \\
\hline slope $\pm \sigma$ & $-1.64 \pm 0.97$ & $-1.86 \pm 1.94$ & $-1.63 \pm 0.89$ & $-1.76 \pm 0.97$ & - & - & $-2.66 \pm 0.43$ & - & - \\
\hline $\mathrm{rms}$ & 0.12 & 0.11 & 0.12 & 0.12 & - & - & - & - & - \\
\hline
\end{tabular}

Notes. ${ }^{a}$ Adopting for V3468 the period found from our data $(P=0.2733 \mathrm{~d}) ;{ }^{b}$ Adopting for V3468 the period from K95 $(P=0.2938 \mathrm{~d})$.

$\sigma=0.06 \mathrm{mag}$, from Muraveva et al. (2018b) calibrated on the same five Galactic RRLs but using their TGAS and DR2 parallaxes.

For RRab only:

$M_{[3.6]}=-2.370( \pm 0.139) \log (P)-1.181$

from Neeley et al. (2015);

$M_{[3.6]}=-2.342( \pm 0.140) \log (P)-1.155( \pm 0.089)$

$\sigma=0.040$ mag, from Neeley et al. (2017).

The Neeley et al. (2017) relations are from a reanalysis of the M4 data in Neeley et al. (2015) using the S19.2 Spitzer pipeline reduced data, combined with $H S T$ parallaxes for zero-point calibration. We include both the old and new Neeley et al. relations here for completeness and for consistency with upcoming SMHASH publications. The PL relations of Muraveva et al. (2018b) are derived for RRc+RRd+RRab whose data were processed with the same Spitzer pipeline as in Neeley et al. (2015). Consistently with the other midIR PL relations considered here, the zero point of Muraveva et al. (2018b) PL relation in equation (5) is calibrated on the HST parallaxes while the zero point in equation (6) on the same five Galactic RRLs of Benedict et al. (2011) but using Gaia DR2 parallaxes for four stars and TGAS parallax for one star (RR Lyr).

Figs 4-6 show the agreement between the PLs published in Madore et al. (2013), Neeley et al. (2015), Neeley et al. (2017), and Muraveva et al. (2018b) (equations 2, 3, 4, 5 and 6, respectively) and our data within $2 \sigma$. Only the D1 and D4 subsamples are shown in the figures, but results for all four subsamples are summarized in Table 4, where our measured RRab+RRc and RRab-only slopes from each data set are shown to be compatible within the respective errors with those from Madore et al. (2013), Neeley et al. (2015), Neeley et al. (2017), and Muraveva et al. (2018b). The results from the D4 data set are particularly encouraging. The RRab-only and 


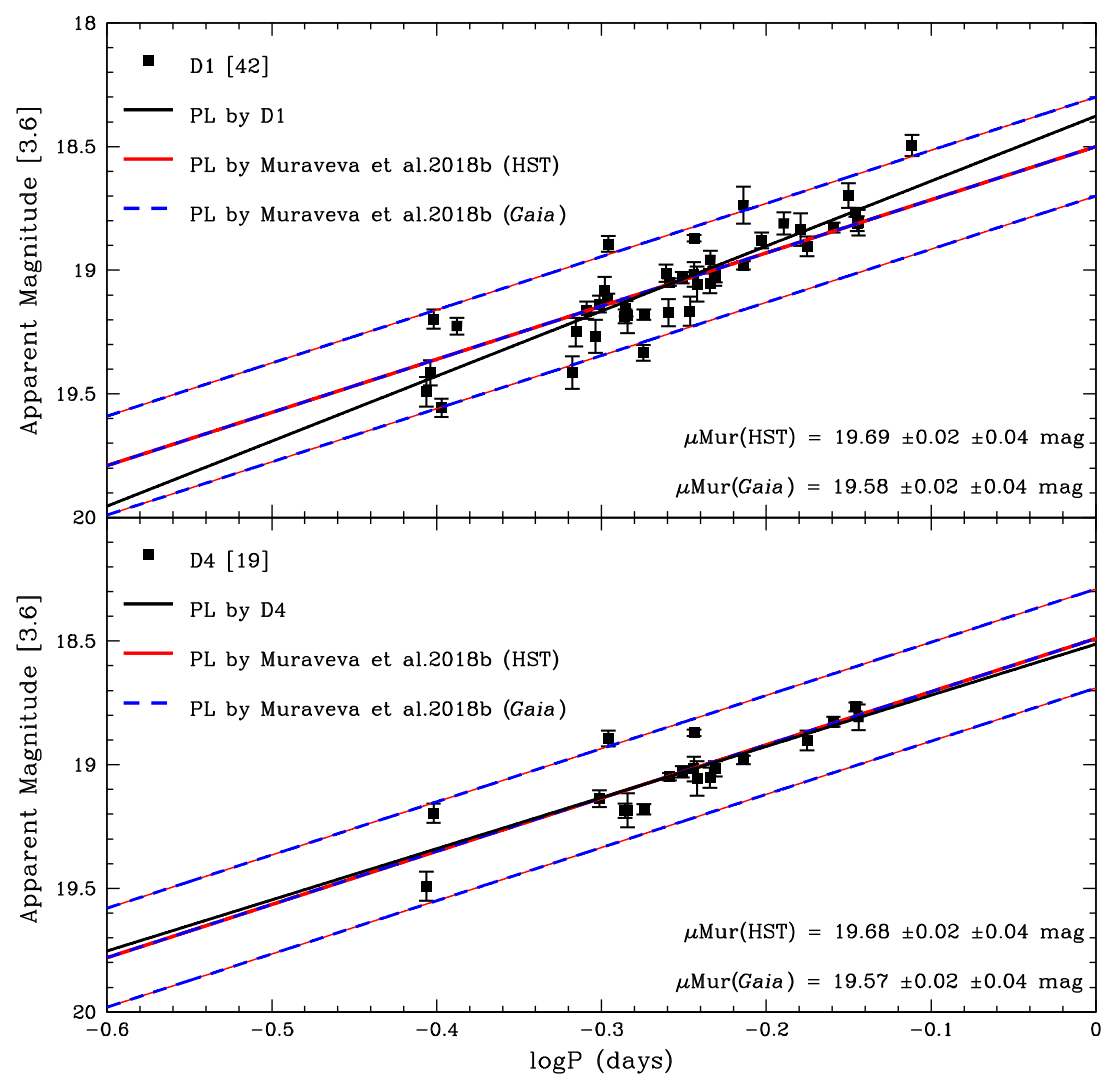

Figure 6. $3.6 \mu \mathrm{m}$ PL relations defined by RRab+RRc stars in the D1 and D4 samples (black solid lines), compared with the PL relations by Muraveva et al. (2018b) calibrated on HST and Gaia parallaxes (thick solid red and dashed blue lines, respectively). The periods of the RRc stars have been fundamentalized.

RRab+RRc slopes derived using this subsample agree within the uncertainties with those from Neeley et al. and Madore et al. (2013). The agreement of our RRab+RRc slope with that of Muraveva et al. (2018b) is particularly remarkable. Although D4 is a small sample, we are confident that it is free from any contaminating photometric effects. The slopes derived from the four samples all have values in the region predicted from other multiwavelength analyses (see fig. 8 in Neeley et al. 2015, and an updated version of fig. 4 in Madore et al. 2013). This adds further weight to the semi-empirical conclusion that the slopes of RRL PLs in the mid-IR bands are well constrained in a range from -2.2 to -2.6 . The confirmation that our PL slopes are in agreement with published values is an important test of our photometry and of the universality of the RRL PL slope. We adopt the PL slopes from the literature to measure the distance modulus of Sculptor because (a) they are more robustly measured than is possible with our data set and (b) an independent PL slope measurement allows a more accurate measurement of distance due to fewer free parameters in the fit.

We measured the distance modulus of Sculptor using each of the four subsamples and the various empirical PL relation calibrations finding comparable results for a given fiducial PL ( $\Delta \mu \leq 0.02 \mathrm{mag}$ among data sets). They span the range from $19.55 \mathrm{mag}$ for the Neeley et al. (2017) PL relation applied to the D2 sample, to $19.68 \mathrm{mag}$ for the Madore et al. (2013) PL relations and almost any of the Sculptor samples. The results obtained for each sample of RRabonly and RRab+RRc are in complete agreement within both the statistical and the systematic uncertainties using the Madore et al. (2013), Neeley et al. (2015), Neeley et al. (2017), and Muraveva et al. (2018b) PL relations. We define the statistical error as the rms measured by each data set divided by the square root of the number of RRLs in the data set, $\sigma_{\text {stat }}=r m s / \sqrt{N_{\mathrm{RRL}}}$; the photometric error as the standard deviation of light-curve residuals; and the systematic error for each data set as the propagation of the rms of the reference PL and data set; $\sigma_{\text {sys }}=\sqrt{\left(r m s_{\text {ref }}\right)^{2}+\left(r m s_{\text {dataset }}\right)^{2}}$.

In Table 5, we summarize distance moduli derived using the D1 and D4 data sets and each PL relation calibration. Our preferred values for the Sculptor distance modulus are obtained using the D4 sample along with the Neeley et al. (2017) RRab-only PL relation and the Muraveva et al. (2018b) relations which are valid for $\mathrm{RRab}+\mathrm{RRc}+\mathrm{RRd}$ stars. We consider these PL relations the most accurate as they are the only empirical PL relations derived so far at $3.6 \mu \mathrm{m}$ using time-resolved Warm-IRAC photometry, and are the most applicable to our data. In addition, the relation from $\mathrm{Mu}$ raveva et al. (2018b, equation 6 above) is the first mid-IR PL based on Gaia parallaxes (DR2 and TGAS, for RR Lyr). We find $\mu=$ $19.60 \pm 0.02$ (statistical) \pm 0.04 (photometric) $\operatorname{mag}$ (with $\sigma_{\text {sys }}=$ $0.09 \mathrm{mag}$ ), equivalent to a distance of $83 \pm 1$ (statistical) \pm 2 (photometric) $\mathrm{kpc}$ (with $\sigma_{\text {sys }}=4 \mathrm{kpc}$ ) for Sculptor from Neeley et al. (2017) RRab-only PL relation and $\mu=19.68 \pm 0.02$ (statistical) \pm 0.04 (photometric) mag (with $\sigma_{\text {sys }}=0.11 \mathrm{mag}$ ), equivalent to a distance of $86 \pm 1$ (statistical) \pm 2 (photometric) $\mathrm{kpc}$ (with $\sigma_{\text {sys }}=$ $5 \mathrm{kpc}$ ) and $\mu=19.57 \pm 0.02$ (statistical) \pm 0.04 (photometric) mag (with $\sigma_{\text {sys }}=0.11 \mathrm{mag}$ ), equivalent to a distance of $82 \pm 1$ (statistical) \pm 2 (photometric) $\mathrm{kpc}$ (with $\sigma_{\text {sys }}=5 \mathrm{kpc}$ ) from the Muraveva et al. (2018b) RRab+RRc+RRd PL relations based on HST and Gaia parallaxes, respectively. These results confirm the value found recently in optical bands by Martínez-Vázquez et al. (2015) ( $\mu_{0}=19.62 \pm 0.04$ mag from 290 RRLs).

Considering the Neeley et al. (2017) RRab+RRc PL relation, the distance modulus becomes $\mu=19.56 \pm 0.02$ (statistical) \pm 
Table 5. Distance moduli for Sculptor derived using the D1 and D4 RR Lyrae samples and different empirical relations.

\begin{tabular}{|c|c|c|c|}
\hline Relation & & D1 [42] & D4 [19] \\
\hline Madore et al. (2013) & $\mu$ & 19.68 & 19.68 \\
\hline \multirow[t]{2}{*}{ Neeley et al. (2015) } & $\mu$ & 19.63 & 19.63 \\
\hline & $\sigma_{\text {stat }} \pm \sigma_{\text {phot }}\left(\sigma_{\text {syst }}\right)$ & $0.02 \pm 0.04(0.14)$ & $0.02 \pm 0.04(0.13)$ \\
\hline \multirow[t]{2}{*}{ Neeley et al. (2017) } & $\mu$ & 19.57 & 19.56 \\
\hline & $\sigma_{\text {stat }} \pm \sigma_{\text {phot }}\left(\sigma_{\text {syst }}\right)$ & $0.02 \pm 0.04(0.12)$ & $0.02 \pm 0.04(0.10)$ \\
\hline Muraveva et al. (2018b) (HST) & $\sigma_{\text {stat }} \pm \sigma_{\text {phot }}\left(\sigma_{\text {syst }}\right)$ & $0.02 \pm 0.04(0.12)$ & $0.02 \pm 0.04(0.11)$ \\
\hline \multirow[t]{2}{*}{ Muraveva et al. (2018b) (Gaia) } & $\mu$ & 19.58 & 19.57 \\
\hline & $\begin{array}{c}\sigma_{\text {stat }} \pm \sigma_{\text {phot }}\left(\sigma_{\text {syst }}\right) \\
\text { RRab }\end{array}$ & $0.02 \pm 0.04(0.12)$ & $0.02 \pm 0.04(0.11)$ \\
\hline \multirow[t]{2}{*}{ Madore et al. (2013) } & $\mu$ & 19.68 & 19.68 \\
\hline & $\sigma_{\text {stat }} \pm \sigma_{\text {phot }}\left(\sigma_{\text {syst }}\right)$ & $0.02 \pm 0.04(0.14)$ & $0.02 \pm 0.04(0.14)$ \\
\hline
\end{tabular}

0.04 (photometric) mag (with $\sigma_{\text {sys }}=0.10 \mathrm{mag}$ ), which is in excellent agreement with the distance inferred from the Muraveva et al. (2018b) PL relation using Gaia parallaxes and also agrees with Martínez-Vázquez et al. (2015)'s results within the relative errors.

In the literature, there are many measurements of the distance to Sculptor obtained using different techniques, such as the tip of the red giant branch (TRGB), the luminosity of the horizontal branch (HB), and the RRL. Focussing in particular on distance estimates based on RRLs, results go from the usual optical bands $\left(\mu_{0}=19.71 \mathrm{mag}\right.$ from $226 \mathrm{RRLs}, \mathrm{K} 95 ; \mu_{0}=19.59 \mathrm{mag}$ from 226 RRLs, Tammann et al. 2008; $\mu_{0}=19.68 \pm 0.08$ mag from 78 RRLs, Pietrzyński et al. 2008) to the near-IR bands where Pietrzyński et al. (2008) have measured a distance to Sculptor of $19.67 \pm 0.02 \pm 0.12 \mathrm{mag}$ using 78 RRLs analysed in $J$ and $K$ bands. Fig. 7 summarizes all the distance measurements to Sculptor derived in various works using independent distance indicators and collected in the NASA/IPAC Extragalactic Database (NED). The green orange and cyan star symbols and bars mark the value and photometric uncertainty obtained in this work adopting Neeley et al. (2017)'s and Muraveva et al. (2018b)'s PL relations for the D4 RRab+RRc sample.

The distance that we provide has a statistical uncertainty of 2.4 percent. If we also consider the systematic uncertainty, the total uncertainty increases to 4.8 per cent.

We find that all our results are consistent with the modulus estimation provided by Dolphin (2002) (labelled 13 in Fig. 7) using the colour-magnitude diagram, thanks to the large error bars of this latter study, while Neeley et al. (2017)'s and only Muraveva et al. (2018b)'s relations based on Gaia parallaxes agree with the measurement by Weisz et al. (2014) (labelled 12 in Fig. 7). Our distance moduli are also in good agreement with the values reported by Kaluzny et al. (1995), Rizzi et al. (2007), Pietrzyński et al. (2008), Tammann et al. (2008), Górski et al. (2011), Tully et al. (2013), and Martínez-Vázquez et al. (2015) that employ the tip of the Red Giant Branch (TRGB), different samples of RRLs (RRab-only or RRab+RRc), and Huxor \& Grebel (2015) carbonrich long period variables (LPV) (labelled 2, 9, 8, 7, 3, 4, 6, and 1 in Fig. 7). Compared with Huxor \& Grebel (2015) which used two carbon-rich LPV stars only, our findings using the Muraveva et al. (2018b)'s relations based on HST parallaxes are in agreement within the photometric errors. Our results overlap with Pietrzyński et al. (2008) (point 2, in Fig. 7), who adopted the HB magnitude as their distance indicator but all our measurements are $\sim 0.1-0.2 \mathrm{mag}$ systematically longer than Webbink (1985)'s and $\sim 0.1-0.2 \mathrm{mag}$ systematically shorter than Salaris et al. (2013)'s, who both also used the HB (respectively, points 11 and 10 in Fig. 7). On the other hand, our moduli are always longer than Menzies et al. (2011)'s moduli (labelled 5 in Fig. 7), which were derived using the Miras and the TRGB. This is because their measurements are based on an LMC modulus of $18.39 \mathrm{mag}$. This value is shorter than the most recent and LMC modulus now solidly anchored to $18.493 \pm 0.008$ (stat) mag (with $\sigma_{\text {sys }}=0.047 \mathrm{mag}$ ) by Pietrzyński et al. (2013). Indeed, assuming the latter value as reference, all Menzies et al. (2011) measurements are consistent with our results (red points, in Fig. 7).

\subsection{Metallicity}

In their spectroscopic study of 107 RRLs in Sculptor, C05 found individual metallicities ranging from -2.40 to $-0.85 \mathrm{dex}$, with a mean value of -1.83 and a significant dispersion of $\pm 0.26 \mathrm{dex}$. This large spread in metallicity makes Sculptor an excellent test bed for investigating potential metallicity effects on the RRL PL relations. We have 20 RRLs (18 RRab, 2 RRc) in common with the C05 catalogue (DZ sample, Table 3 ), covering a metallicity range from -2.31 to -1.38 dex (see Table 1 , column 11). This range gives a $\Delta[\mathrm{Fe} / \mathrm{H}]=$ 0.97 dex that is smaller than the 1.55 dex value corresponding to the C05 whole sample, but still wide enough to make considerations on PLs metallicity effects. In their combined photometric and spectroscopic study, Tolstoy et al. (2004) confirmed what was found by Majewski et al. (1999) only on photometric grounds, i.e. that Sculptor contains two distinct stellar populations: metal-poor stars with $[\mathrm{Fe} / \mathrm{H}]_{\mathrm{ZW}}<-1.7$ dex and metal-rich stars with $[\mathrm{Fe} / \mathrm{H}]_{\mathrm{ZW}}>$ -1.7 dex. This was confirmed in further complementary studies (for example, de Boer et al. 2011; Breddels \& Helmi 2014). Following the Tolstoy et al. (2004) scheme, we now split our sample of 20 RRLs with individual spectroscopic metallicities from $\mathrm{C} 05$ into two groups: metal-poor stars with $[\mathrm{Fe} / \mathrm{H}]_{\mathrm{ZW}}<-1.7 \operatorname{dex}(N=14$ stars) and metal-rich stars with $[\mathrm{Fe} / \mathrm{H}]_{\mathrm{ZW}}>-1.7 \mathrm{dex}(N=6$ stars $)$.

We show the PL relations for these two samples, with the fits defined using the metal-rich sample in the top panel and the 


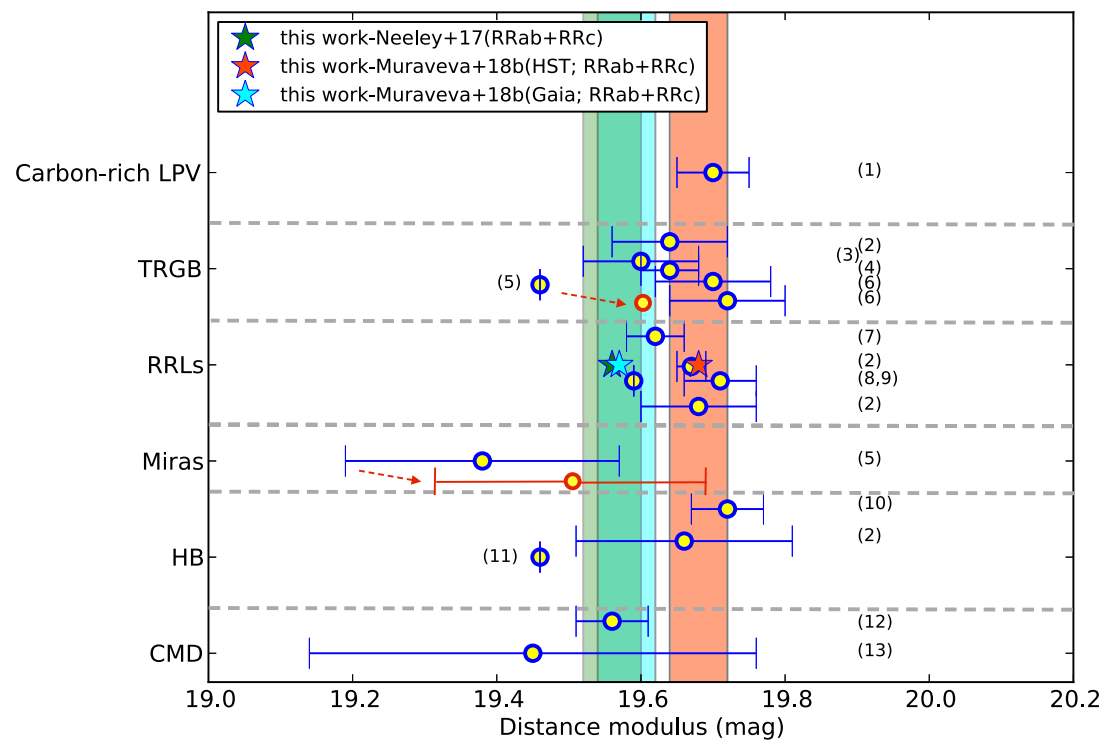

Figure 7. Sculptor distance modulus estimations derived by different techniques. The green, orange, and cyan star symbols and bars mark the values and photometric uncertainties obtained in this work adopting, respectively, the Neeley et al. (2017)'s and Muraveva et al. (2018b)'s PL relations for the D4 RRab+RRc sample. (1) Huxor \& Grebel (2015); (2) Pietrzyński et al. (2008); (3) Tully et al. (2013); (4) Rizzi et al. (2007); (5) Menzies et al. (2011); (6) Górski, Pietrzyński \& Gieren (2011); (7) Martínez-Vázquez et al. (2015); (8) Kaluzny et al. (1995); (9) Tammann, Sandage \& Reindl (2008); (10) Salaris et al. (2013); (11) Webbink (1985); (12) Weisz et al. (2014); (13) Dolphin (2002).

metal-poor sample in the bottom panel (solid black lines) comparing with Madore et al. (2013), Neeley et al. (2015), and Neeley et al. (2017) in Fig. 8. Fig. 9 is the same as Fig. 8, but compared with the PL relations in Muraveva et al. (2018b). Using the Neeley et al. (2015) PL relation to determine distance moduli, we find $\mu=$ $19.61 \mathrm{mag}\left(\sigma_{\text {stat }}=0.05 \mathrm{mag}\right)$ for metal-rich stars and $\mu=19.63 \mathrm{mag}$ $\left(\sigma_{\text {stat }}=0.02 \mathrm{mag}\right)$ for metal-poor stars, in perfect agreement with each other within their respective errors. If we also consider the Madore et al. (2013) PL relations, we obtain $\mu=19.66 \pm 0.05$ and $19.68 \pm 0.02 \mathrm{mag}$ for metal-rich and metal-poor stars, respectively. Adopting the PL relations from Neeley et al. (2017) gives $\mu=19.55 \pm 0.05 \mathrm{mag}$ for metal-rich and $\mu=19.58 \pm 0.02 \mathrm{mag}$ for metal-poor stars. Finally, using Muraveva et al. (2018b) PL relations calibrated on HST parallaxes gives $\mu=19.67 \pm 0.05$ and $19.70 \pm 0.02$ mag for metal-rich and metal-poor stars, respectively. These same relations calibrated on Gaia parallaxes give $\mu=$ $19.56 \pm 0.05$ and $19.59 \pm 0.02$ mag for metal-rich and metal-poor stars, respectively. These results suggest that the metallicity dependence, if any, should be very small (Table 6).

Taking into account the individual spectroscopic metallicity measured for the DZ sample, we used the PLZ reported in table 3 of Neeley et al. (2017) to calculate the Sculptor distance for metal-poor and metal-rich stars, getting $\mu=19.63 \pm 0.02$ (stat) \pm 0.04 (phot) mag (with $\sigma_{\text {sys }}=0.11 \mathrm{mag}$ ) and $\mu=19.52 \pm 0.05$ (stat) \pm 0.03 (phot) mag (with $\sigma_{\text {sys }}=0.13 \mathrm{mag}$ ), respectively, and $\mu=19.59 \pm 0.03$ (stat) \pm 0.04 (phot) mag (with $\sigma_{\text {sys }}=0.12 \mathrm{mag}$ ) for the whole DZ sample.

Again these distance moduli, in agreement within the systematic errors with the values derived in the previous section, confirm the agreement between empirical and theoretical results.

Admittedly, the number of RRLs with spectroscopic metallicity in our sample is rather small, corresponding to less than a half of our total sample (42 RRLs) and less than 1/10 of the K95 sample (226 RRLs). Furthermore, as it is well known, the RRL population in classical dSphs such as Sculptor does not conform to the Ooster- hoff dichotomy observed in the Galactic globular clusters (GGCs), but rather has properties intermediate between the two Oosterhoff types $^{7}$ (see e.g. table 3 of Clementini 2010). Hence, the periodamplitude (Bailey) diagram cannot be used to infer an approximate indication of any metallicity spread for the RRLs in our D4 sample that do not have spectroscopic measurements available.

This is clearly shown by Fig. 10, which in the left-hand panel reports the $V$-band period-amplitude (Bailey) diagram of the 42 RRLs in our sample, based on the periods and $V$ amplitudes reported in K95 and C05 (Table 1, columns 5 and 8, respectively). The grey lines in the left-hand panel define the loci of Oosterhoff properties according to Clement \& Rowe (2000), separating the plane into the Oo I (solid line) and Oo II (dashed line) regions. There is no sign of a separation between the two Oo types and consequently between the two populations with different metallicities defined by Tolstoy et al. (2004). Moreover, if we separate the RRLs whose metallicities are known from the spectroscopic study of C05 into metal-poor and metal-rich samples (magenta hexagons and green four-pointed stars, respectively, in Fig. 10), they do not show any significant bimodality.

The Oosterhoff-intermediate nature of Sculptor was clearly established by K95 based on the pulsation properties of over 200 RRLs detected in the galaxy, for which these authors derived

\footnotetext{
${ }^{7}$ In the Milky Way the GGCs separate into two distinct groups or Oosterhoff types according to the pulsation properties of their RR Lyrae populations: Oosterhoff type I (Oo I) clusters have a mean period of the RRab variables, $<P_{a b}>, \sim 0.55 \mathrm{~d}$ and the frequency of RRc stars over the total number of RRLs: $f_{c}=N_{\mathrm{RRc}} /\left(N_{\mathrm{RRab}}+N_{\mathrm{RRc}}\right) \sim 0.17$, while Oo II systems have $\left\langle P_{a b}\right\rangle$ $\sim 0.65 \mathrm{~d}$ and $f c \sim 0.44$ (Clement \& Rowe 2000). Differences in the mean period of the RRc variables are also found between the two groups, $\left\langle P_{c}\right\rangle$ $\sim 0.32$ and $\sim 0.37 \mathrm{~d}$ for Oo I and Oo II types, respectively (see e.g. Catelan 2009). The Oosterhoff dichotomy of the MW GGC also reflects a difference in metal abundance by which Oo I clusters generally are more metal-rich having $[\mathrm{Fe} / \mathrm{H}] \geq-1.5$ dex and Oo II clusters generally are metal-poor having $[\mathrm{Fe} / \mathrm{H}] \simeq-2 \mathrm{dex}$.
} 


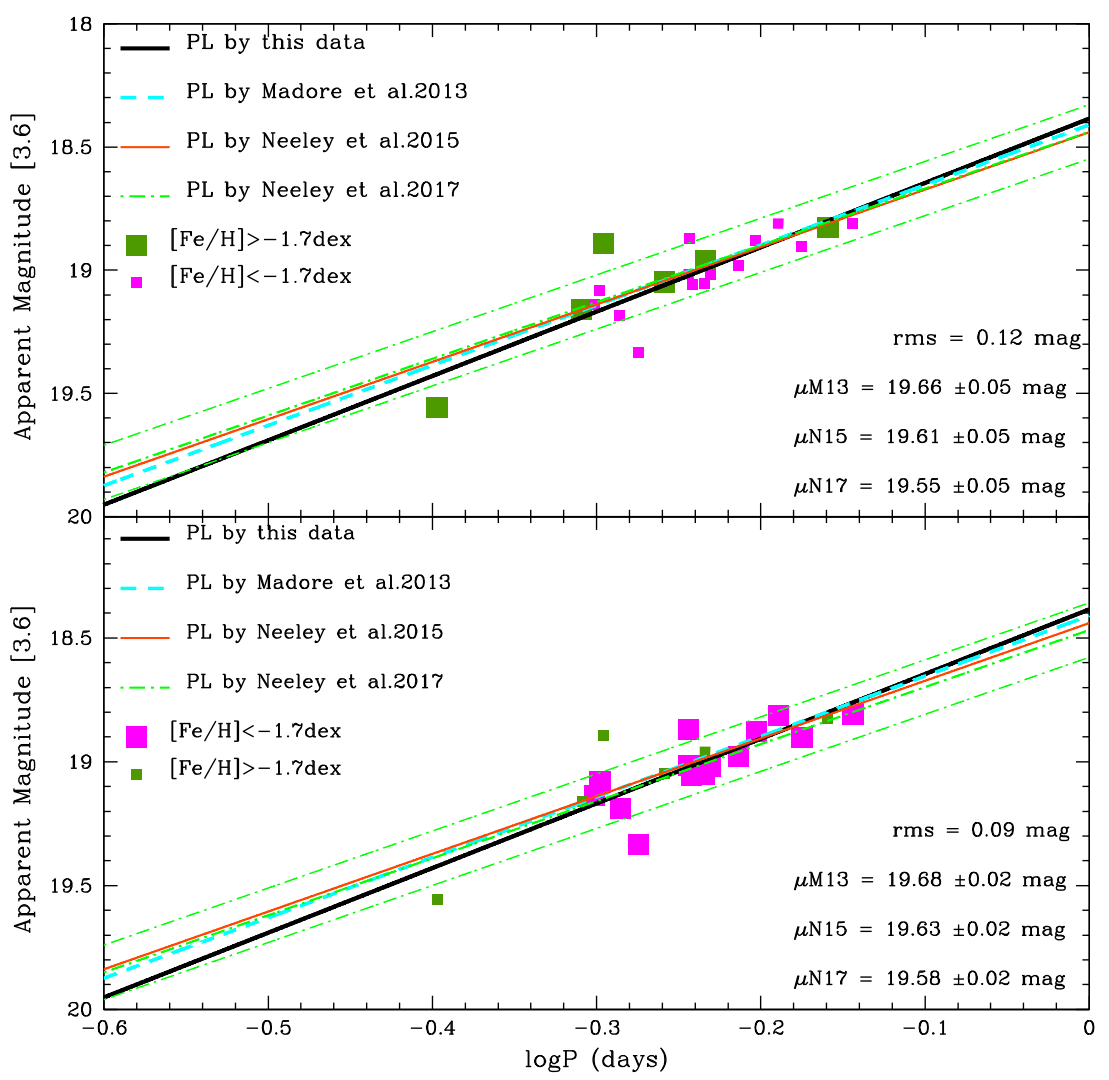

Figure 8. PL relations for the only 20 RRLs (DZ sample) whose spectroscopic metallicities are known from C05. Green and magenta filled squares mark metal-rich and metal-poor RRL, respectively. The black solid line represents the least-squares fit to the metal-rich (top panel) and metal-poor stars (bottom panel), respectively. The green (dash-dotted) lines indicate the Neeley et al. (2017) PL relation $\pm 2 \sigma$ deviation. Similarly, the cyan (dashed) and orange (thin solid) lines mark the Madore et al. (2013) and the Neeley et al. (2015) PL relations along with their $\pm 2 \sigma$ deviations. The periods of the RRc stars have been fundamentalized.

$\left\langle P_{a b}\right\rangle=0.587 \mathrm{~d}$ and $f c=N_{\mathrm{RRc}} /\left(N_{\mathrm{RRab}}+N_{\mathrm{RRc}}\right)=0.40$. We reach the same conclusions here if we consider the average pulsation properties of our data sets shown in Fig. 10. Table 7 lists $f c,\left\langle P_{a b}\right\rangle$, and $\left.<P_{c}\right\rangle$ values calculated for each data set: D1, D4, and DZ (split into metal-poor and metal-rich subsamples) along with K95 values for a comparison. We find, on the one hand, that the $f c$ and $\left\langle P_{c}\right\rangle$ values suggest an Oo I classification for all data sets. On the other hand, $<P_{a b}>$ places them in an intermediate classification between Oo I and Oo II. Moreover, for all samples, the standard deviation related to the mean period of RRab stars is rather large $(\sigma \sim 0.07 \mathrm{~d})$, which indicates a mixing of both metal-poor and metal-rich components among Sculptor RRLs that remain indistinguishable irrespective of pulsation properties.

The right-hand panel of Fig. 10 shows instead the mid-IR Bailey diagram based on our light curves at $3.6 \mu \mathrm{m}$ with the same symbols and colours coding as in the left-hand panel. The RRL distribution is broader in this plane at longer wavelengths than in the $V$ band and it is also much broader than the period-amplitude diagram at $3.6 \mu \mathrm{m}$ obtained by Muraveva et al. (2018b) for the RRL in the Reticulum cluster (see upper panel of fig. 6 in that paper).

As a further test, Fig. 11 shows the individual PL derived distance moduli versus metallicity for each of the 20 RRLs with a spectroscopic metallicity (DZ sample) and using as a reference the PL relation derived by Neeley et al. (2017) and only the RRab stars in the D4 sample. However, we note that results do not change if a different fiducial PL is adopted. The least-squares fit (black line) has been calculated for four different cases: Case A - using the whole sample, case B - removing only V1932, the star with the shortest derived distance modulus $(\mu=19.384 \pm 0.035 \mathrm{mag})$, case $\mathrm{C}$ - removing only V1546, the star with the longest derived distance modulus ( $\mu=19.873 \pm 0.032 \mathrm{mag}$ ), and case $\mathrm{D}-$ excluding both V1932 and V1546. The resultant fits are summarized in Table 8. In each case, the slopes $(\mathrm{d} \mu / \mathrm{d}[\mathrm{Fe} / \mathrm{H}])$ are consistent with zero within $1 \sigma$. Again, this strengthens the evidence that any metallicity effect, if it exists, is negligible in our sample. Indeed, a direct correlation between distance modulus and metallicity is not observable in our data, neither in this test nor in the analysis for Figs 8 and 9 where we looked specifically for differences in the PL relations derived from known metal-rich and metal-poor stars.

\section{SUMMARY AND CONCLUSION}

As part of the SMHASH program, using IRAC-Spitzer data we have determined a new distance modulus of the Sculptor dSph using RRLs located in the inner region of the galaxy. We obtained time-series photometry for 49 RRLs, (36 RRab and $13 \mathrm{RRc}$ ) at $3.6 \mu \mathrm{m}$. Seven stars were discarded from the initial sample mainly due to photometric contamination effects. Adopting periods from Kaluzny et al. (1995) and Clementini et al. (2005), we built light curves for the remaining 42 highest quality stars, delineating their mid-IR pulsation properties. In order to investigate the photometric properties of the sample, and to choose the highest quality subsample of stars, we created four data sets (D1, D2, D3, and D4) by removing various problematical stars (see Section 3 for details). 


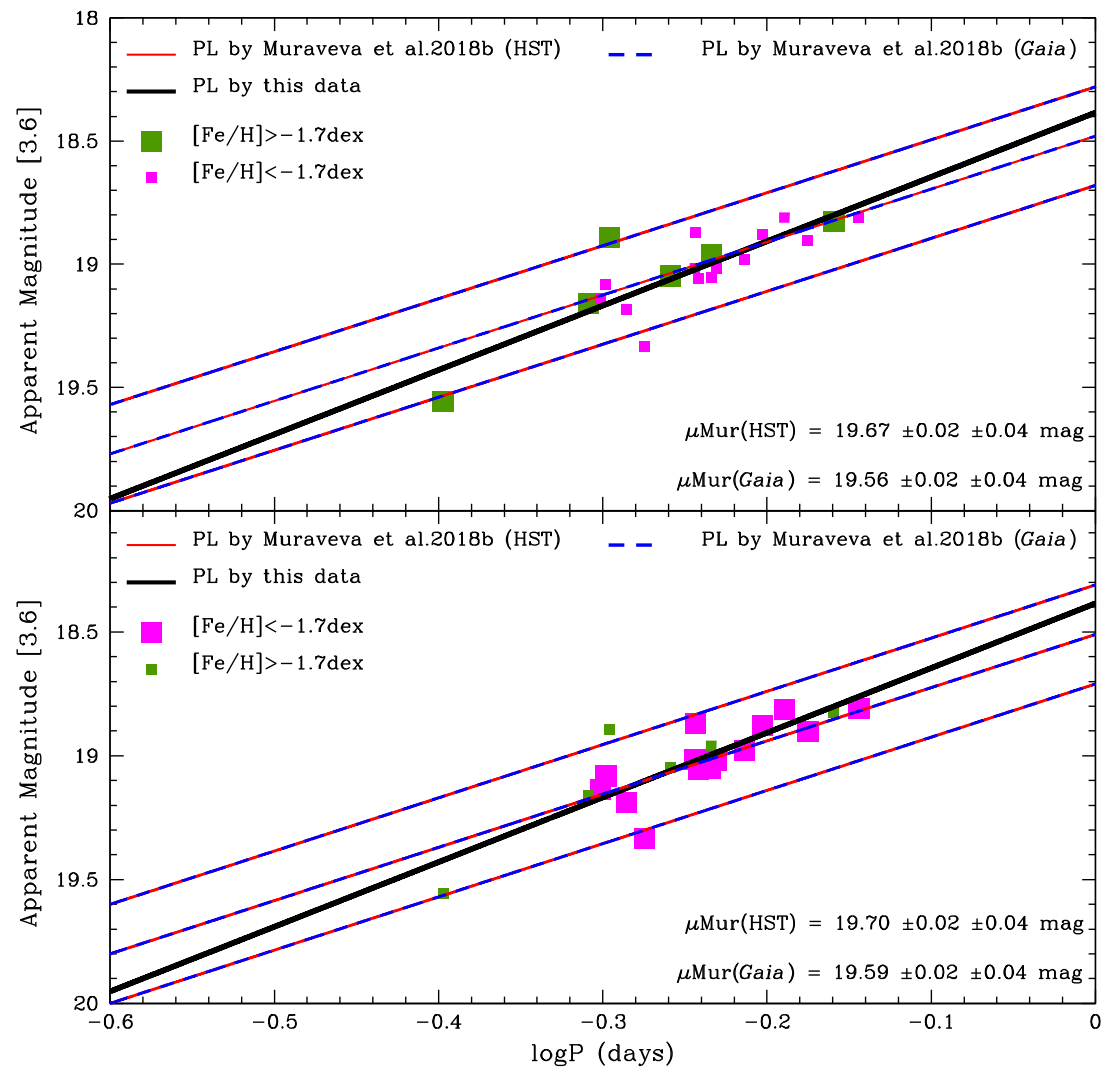

Figure 9. Same as in Fig. 8 but with the thick solid red and the dashed blue lines showing the PL relations by Muraveva et al. (2018b), calibrated, respectively, on the HST and Gaia parallaxes, $\pm 2 \sigma$ deviation. The periods of the RRc stars have been fundamentalized.

Table 6. Distance moduli for Sculptor derived using different DZ subsamples and different empirical PL relations.

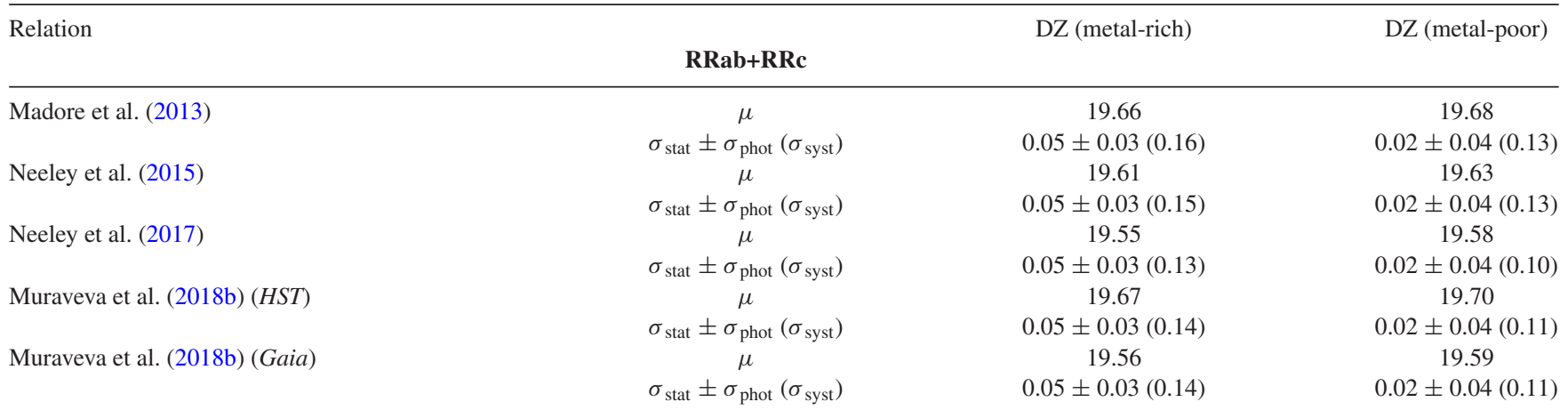

Notes. Metal-rich are RRLs with $[\mathrm{Fe} / \mathrm{H}]>-1.7 \mathrm{dex}\left(N_{\mathrm{RRL}}=6\right) ;$ metal-poor are RRLs with $[\mathrm{Fe} / \mathrm{H}]<-1.7 \mathrm{dex}\left(N_{\mathrm{RRL}}=14\right)$.

PL relations were derived for each RRab-only and RRab+RRc (fundamentalized) data set, and were found to be in reasonable agreement (i.e. within $1 \sigma$ ) with the empirical relations published in Madore et al. (2013) and Neeley et al. (2015), as well as the revised relations from Neeley et al. (2017) and the recent PL relations derived by Muraveva et al. (2018b) (Table 4). We adopt for Sculptor the distance modulus derived from the D4 sample, as this has the cleanest RRL selection and the best-fitting slopes are the closest to the published empirical relations that we have considered as a reference in this work. We are aware that mid-IR studies of the RRL PL are increasing (Madore et al. 2013; Dambis et al. 2014; Klein et al. 2014; Neeley et al. 2015, 2017; Muraveva et al. 2018b), but choose the Madore et al. (2013), Neeley et al. (2015), Neeley et al. (2017), and Muraveva et al. (2018b) PLs as fiducial as they are built using the sample of RRL calibrators whose trigonometric parallaxes were measured by Benedict et al. (2011) with the FGS@HST and, more recently, by Gaia. Furthermore, because Neeley et al. (2015) and Muraveva et al. (2018b) derived PL relations using Warm IRAC-Spitzer data, the same instrument, and passband used for the work here, we consider them the most reliable reference for our study.

Due to the significant metallicity spread observed in the Sculptor's RRL and the presence of two separate stellar populations (Majewski et al. 1999; Tolstoy et al. 2004), we also investigated the potential for metallicity effects on the mid-IR RRL PL relation and our subsequent Sculptor distance determination. We considered a sample (DZ) containing 20 RRLs for which Clementini et al. (2005) provided spectroscopic metallicity measurements. In addition, we split the DZ sample into two further subsamples - metal-poor $\left([\mathrm{Fe} / \mathrm{H}]_{\mathrm{ZW}}<-1.7\right)$ and metal-rich $\left([\mathrm{Fe} / \mathrm{H}]_{\mathrm{ZW}}>-1.7\right)$ - reflecting 

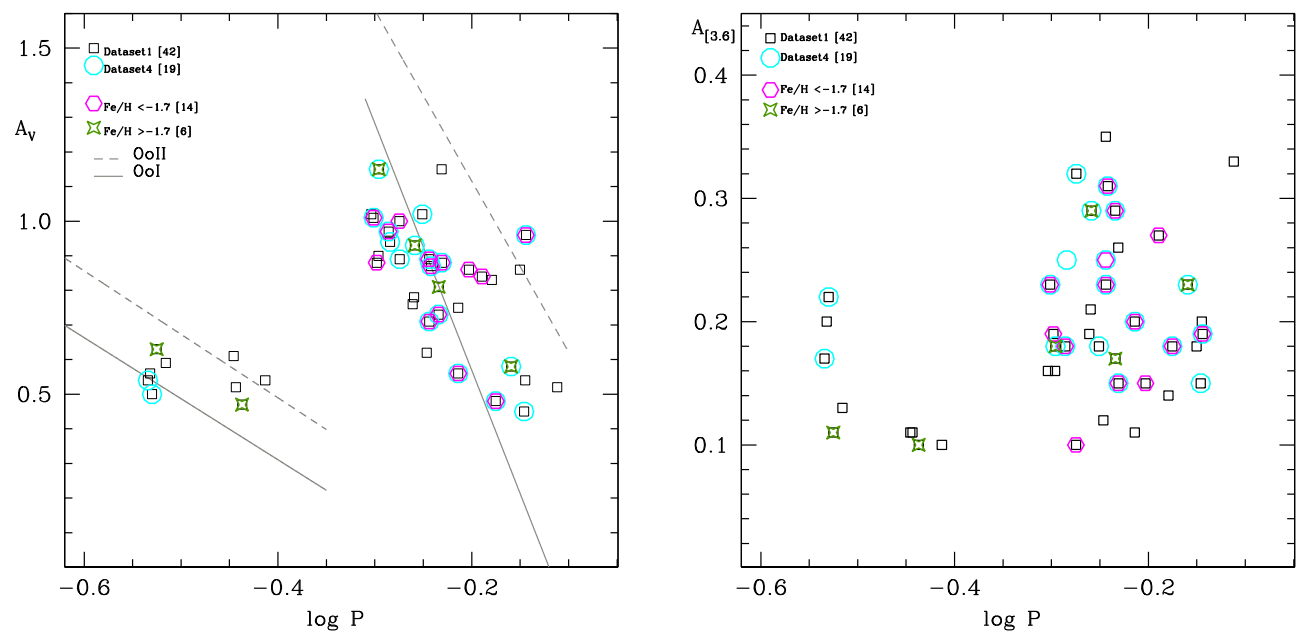

Figure 10. Left: $V$-band period-amplitude diagram for the 42 RRLs of Sculptor, periods and amplitudes are from K95 and C05. The grey lines mark the loci of the OoI (solid) and OoII (dashed) Galactic GCs from Clement \& Rowe (2000), where, typically, OoI systems are more metal-rich $([\mathrm{Fe} / \mathrm{H}] \sim-1.5 \mathrm{dex})$ than the OoII systems $([\mathrm{Fe} / \mathrm{H}] \sim-2 \mathrm{dex})$. Black empty squares are the D1 sample while cyan circles are the D4 sample. Magenta hexagons and green four-pointed stars denote metal-poor and metal-rich samples, respectively, according to C05 metallicities. Right: Mid-IR period-amplitude diagram, adopting the amplitudes at $3.6 \mu \mathrm{m}$ derived in this study. Colours and symbols as on the left-hand panel.

Table 7. Pulsation properties of each of our data sets compared with K95 RRL sample.

\begin{tabular}{lccc}
\hline Sample & $\left\langle P_{a b}\right\rangle$ & $f c$ & $<P_{c}>$ \\
\hline D1 & $0.594 \pm 0.075$ & 0.21 & $0.328 \pm 0.036$ \\
D4 & $0.587 \pm 0.069$ & 0.10 & $0.294 \pm 0.001$ \\
DZ & $0.586 \pm 0.063$ & 0.11 & $0.332 \pm 0.034$ \\
DZ $^{a}$ & $0.586 \pm 0.061$ & - & - \\
DZ $^{b}$ & $0.586 \pm 0.063$ & 0.33 & $0.332 \pm 0.034$ \\
K95 $^{b}$ & $0.587 \pm 0.081$ & 0.40 & $0.336 \pm 0.041$
\end{tabular}

Notes. ${ }^{a}$ Sample contains 14 RRLs defined as a metal-poor subsample.

${ }^{b}$ Sample contains six RRLs defined as a metal-rich subsample.

the two populations found by Tolstoy et al. (2004). Using these subsamples to remeasure the PL slope, and making comparisons both between the different PLs and distance moduli measured in our work and adopting the slopes from Madore et al. (2013), Neeley et al. (2015), Neeley et al. (2017), and Muraveva et al. (2018b), we do not find any evidence for a significant metallicity effect on our result.

We measure the distance modulus of Sculptor as $\mu=$ $19.60 \pm 0.02$ (statistical) \pm 0.04 (photometric) $\operatorname{mag}$ (with $\sigma_{\text {sys }}=$ $0.09 \mathrm{mag}$ ), corresponding to $83 \pm 1$ (statistical) \pm 2 (photometric) $\mathrm{kpc}$ (with $\sigma_{\text {sys }}=4 \mathrm{kpc}$ ), using the 17 RRab stars of the D4 sample and adopting as fiducial the $3.6 \mu \mathrm{m}$ empirical PL relation for only RRab stars in the Galactic globular cluster M4 derived by Neeley et al. (2017), or $\mu=19.57 \pm 0.02$ (statistical) \pm 0.04 (photometric) mag (with $\sigma_{\text {sys }}=0.11 \mathrm{mag}$ ) using the whole D4 sample (19 RRL) and the empirical PL relation at 3.6 $\mu \mathrm{m}$ for RRab+RRc+RRd stars in the LMC globular cluster Reticulum recently derived by Muraveva et al. (2018b) calibrated on Gaia parallaxes. We find consistent results for the distance modulus using also the Madore et al. (2013) and Neeley et al. (2015) relations (Table 5). These distances are also in good agreement with the estimates by Tammann et al. (2008), Pietrzyński et al. (2008), and Martínez-Vázquez et al. (2015).

We have also tried to quantify the depth effect set by our data and whether it can affect our distance estimation. The line-of-sight depth can be measured by subtracting in quadrature the distance scatter we found for Sculptor adopting the D4 sample and the dis- tance scatter that Muraveva et al. (2018b) provide for Reticulum: $\left[(0.08)^{2}-(0.06)^{2}\right]^{1 / 2} \simeq 0.05 \mathrm{mag}$, corresponding to $\pm 2 \mathrm{kpc}$, which is completely within our photometric error.

A significant advantage of our study is that we are able to obtain a precise distance estimate of comparable accuracy to the larger studies using an RRL sample that is less than 10 per cent of the size analysed by Martínez-Vázquez et al. (2015) and less than 4 per cent of the total number of Sculptor RRL stars discovered to date. The Sculptor RRL catalogue may not yet be complete, despite having 536 variables identified so far (Martínez-Vázquez et al. 2016b).

The key feature of our study that has enabled this significant leap forward has been moving to the mid-IR to observe RRLs, where (i) the intrinsic dispersion of the RRL PL relation is narrower compared to that at shorter wavelengths, (ii) RRL light curves at $3.6 \mu \mathrm{m}$ have more symmetrical shapes and smaller amplitudes, providing more precise mean magnitudes, and (iii) the effects of reddening/extinction are dramatically reduced. Combined with our confirmation here that any metallicity effect on the $3.6 \mu \mathrm{m}$ PL must be small, if it exists at all, our study of Sculptor sets the stage for our future work on the other dSphs observed in the SMHASH project.

It is undeniable that the error budget of our results is dominated by the systematic error affecting the absolute zero-point calibration of the RRL mid-IR PL relations. Indeed, testing the quality of our mid-IR photometry using different RRL subsamples, we found very similar distance moduli, even identical in many cases, for a given fiducial PL relation, proving that the accuracy of the final distance is not limited by the quality of mid-IR data but rather by the choice of the adopted fiducial PL relation.

In this SMHASH project great contribution is expected from exploitation of Gaia DR2 and future data released from this mission. Gaia DR2 contains a first mapping of full-sky RRL (Holl et al. 2018, Clementini et al. 2018) and parallaxes based on Gaia-only measurements for about 1.3 billion sources (Gaia Collaboration 2018; Lindegren et al. 2018). Among them is a much larger number of Galactic RRLs than the five calibrators with HST parallaxes of Benedict et al. (2011). Muraveva et al. (2018c) have recently derived a new RRL PLZ relation whose slope and zero point are based on the Gaia DR2 parallaxes of about 400 Galactic RRLs. Muraveva 

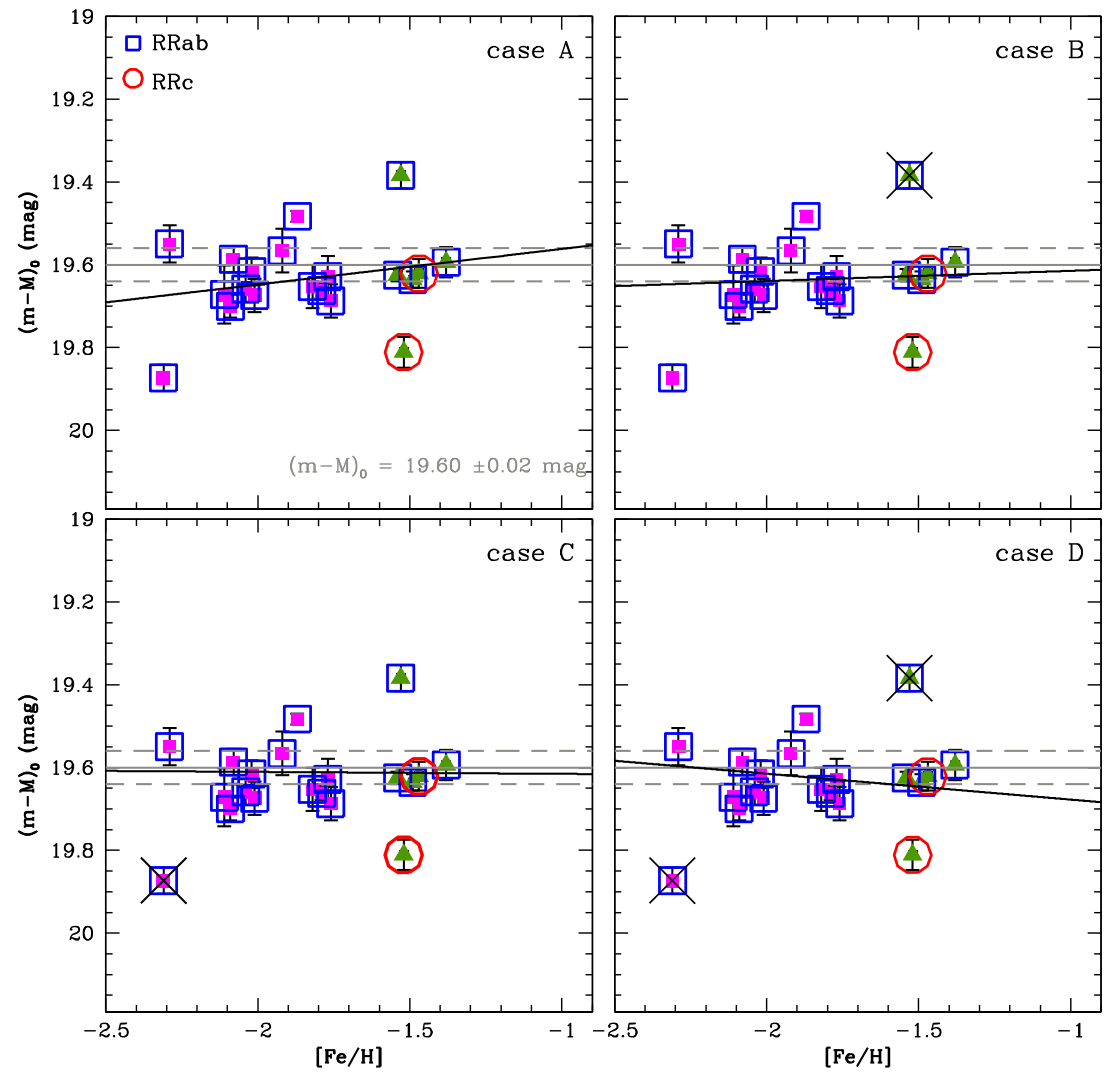

Figure 11. Distance moduli for the 20 RRLs in our DZ sample as a function of $[\mathrm{Fe} / \mathrm{H}]$ (spectroscopic metallicities from C05). The solid grey line is the distance modulus $\pm 2 \sigma$ (dashed lines) estimated using as a reference the PL relation derived by Neeley et al. (2017) and only the RRab stars in the D4 sample. Metal-poor sources are magenta squares and metal-rich are green triangles. RRab and RRc stars are encircled by blue squares and red circles, respectively. The RRLs marked with a cross are the rejected stars. The least-squares fit (black line) has been calculated in four different cases: considering the whole sample (case A, top left panel), removing the 'closest' star (in terms of distance from us; top right panel, case B), removing the 'most distant' star (case C, bottom left panel), and excluding both (case $\mathrm{D}$, bottom right panel). In every case, the slope $(\mathrm{d} \mu / \mathrm{d}[\mathrm{Fe} / \mathrm{H}])$ is consistent with zero within $1 \sigma$ (see the text and Table 8 for details).

Table 8. $\mathrm{d} \mu / \mathrm{d}[\mathrm{Fe} / \mathrm{H}]$ derived from the DZ sample.

\begin{tabular}{lcc}
\hline Case & $\mathrm{d} \mu / \mathrm{d}[\mathrm{Fe} / \mathrm{H}]$ & $\sigma_{\mathrm{d} \mu / \mathrm{d}[\mathrm{Fe} / \mathrm{H}]}$ \\
\hline $\mathrm{A}$ & -0.086 & \pm 0.084 \\
$\mathrm{~B}$ & 0.009 & \pm 0.081 \\
$\mathrm{C}$ & -0.034 & \pm 0.075 \\
$\mathrm{D}$ & 0.051 & \pm 0.065 \\
\hline
\end{tabular}

et al. (2018c) manuscript is not yet published, therefore we decide to not include results based on the new PLZ in our paper.

\section{ACKNOWLEDGEMENTS}

We thank an anonymous referee for comments and suggestions that helped to improve the manuscript. We thank Stijn Wuyts for his help with this manuscript. This work is based in part on observations made with the Spitzer Space Telescope, which is operated by the Jet Propulsion Laboratory, California Institute of Technology under a contract with NASA. Support for this work was provided by NASA through an award issued by JPL/Caltech. This research has made use of the NASA/IPAC Extragalactic Database (NED) which is operated by the Jet Propulsion Laboratory, California Institute of Technology, under a contract with the National Aeronautics and Space Administration and data from the European Space Agency (ESA) mission Gaia (https://www.cosmos.esa.int/gaia), processed by the Gaia Data Processing and Analysis Consortium (DPAC; https: //www.cosmos.esa.int/web/gaia/dpac/consortium). Funding for the DPAC has been provided by national institutions, in particular the institutions participating in the Gaia Multilateral Agreement. Support to this study has been provided by PRIN-INAF2014, 'EXCALIBUR'S' (P.I. G. Clementini) and by Premiale 2015, 'MITiC' (P.I. B. Garilli). GC thanks the Carnegie Observatories visitor program for support as a science visitor. AG thanks Felice Cusano for useful discussions and Vincenzo Ripepi for help with the selection and preparation of the finding chart for the Spitzer observations of the RRL used in this study.

\section{REFERENCES}

Arenou F. et al., 2018, A\&A, 616, 17

Baker M., Willman B., 2015, AJ, 150, 160

Battaglia G., Helmi A., Tolstoy E., Irwin M., Hill V., Jablonka P., 2008, ApJ, 681, L13

Belokurov V., Koposov S. E., 2016, MNRAS, 456, 602

Benedict G. F. et al., 2011, AJ, 142, 187

Blažko S., 1907, Astron. Nachr., 175, 325

Bono G., Caputo F., Castellani V., Marconi M., Storm J., 2001, MNRAS, 326,1183

Bono G., Caputo F., Castellani V., Marconi M., Storm J., Degl'Innocenti S., 2003, MNRAS, 344, 1097 
Braga V. F. et al., 2015, ApJ, 799, 165

Breddels M. A., Helmi A., 2014, ApJ, 791, L3

Bullock J. S., Johnston K. V., 2005, ApJ, 635, 931

Bullock J. S., Kravtsov A. V., Weinberg D. H., 2001, ApJ, 548, 33

Carretta E., Gratton R. G., 1997, A\&AS, 121, 95

Carretta E., Bragaglia A., Gratton R., D’Orazi V., Lucatello S., 2009, A\&A, 508,695

Catelan M., 2009, Ap\&SS, 320, 261

Catelan M., Pritzl B. J., Smith H. A., 2004, ApJS, 154, 633

Clement C. M., Rowe J., 2000, AJ, 120, 2579

Clementini G., 2010, in Sterken C., Samus N., Szabados L., eds, Variable Stars, the Galactic halo and Galaxy Formation. Sternberg Astronomical Institute of Moscow Univ., Moscow, p. 107

Clementini G. et al., 2000, AJ, 120, 2054

Clementini G., Ripepi V., Bragaglia A., Martinez Fiorenzano A. F., Held E. V., Gratton R. G., 2005, MNRAS, 363, 734 (C05)

Clementini G. et al., 2018, A\&A, preprint (arXiv:1805.02079)

Coppola G. et al., 2011, MNRAS, 416, 1056

Dall'Ora M. et al., 2004, ApJ, 610, 269

Dall'Ora M. et al., 2006, ApJ, 653, L109

Dambis A. K., Rastorguev A. S., Zabolotskikh M. V., 2014, MNRAS, 439, 3765

de Boer T. J. L. et al., 2011, A\&A, 528, A119

Dolphin A. E., 2002, MNRAS, 332, 91

Drake A. J. et al., 2013, ApJ, 763, 32

Fazio G. G. et al., 2004, ApJS, 154, 10

Freedman W. L., Madore B. F., Scowcroft V., Burns C., Monson A., Persson S. E., Seibert M., Rigby J., 2012, ApJ, 758, 24

Gaia Collaboration, 2017, A\&A, 605, A79

Gaia Collaboration, 2018, A\&A, 616, A1

Garofalo A. et al., 2013, ApJ, 767, 62

Gavrilchenko T., Klein C. R., Bloom J. S., Richards J. W., 2014, MNRAS, 441,715

Geisler D., Smith V. V., Wallerstein G., Gonzalez G., Charbonnel C., 2005, AJ, 129, 1428

Górski M., Pietrzyński G., Gieren W., 2011, AJ, 141, 194

Gran F. et al., 2016, A\&A, 591, A145

Hendel D. et al., 2017, MNRAS, 479, 570

Holl B. et al., 2018, A\&A, preprint (arXiv:1804.09373)

Huxor A. P., Grebel E. K., 2015, MNRAS, 453, 2653

Iben I., Jr., 1974, ARA\&A, 12, 215

Jacyszyn-Dobrzeniecka A. M. et al., 2016, Acta Astron., 67, 1

Johnston K. et al., 2013, Spitzer Proposal, 10015

Kaluzny J., Kubiak M., Szymanski M., Udalski A., Krzeminski W., Mateo M., 1995, A\&AS, 112, 407 (K95)

Klein C. R., Richards J. W., Butler N. R., Bloom J. S., 2014, MNRAS, 440, L96

Kovács G., 2001, A\&A, 375, 469

Lindegren L. et al., 2016, A\&A, 595, A4

Lindegren L. et al., 2018, A\&A, 616, A2

Longmore A. J., Fernley J. A., Jameson R. F., 1986, MNRAS, 220, 279

Longmore A. J., Dixon R., Skillen I., Jameson R. F., Fernley J. A., 1990, MNRAS, 247, 684

Madore B. F. et al., 2013, ApJ, 776, 135

Majewski S. R., Siegel M. H., Patterson R. J., Rood R. T., 1999, ApJ, 520, L33

Makovoz D., Khan I., 2005, Astron. Data Anal. Softw. Syst. XIV, 347, 81

Marconi M. et al., 2015, ApJ, 808, 50

Martínez-Vázquez C. E. et al., 2015, MNRAS, 454, 1509

Martínez-Vázquez C. E. et al., 2016a, MNRAS, 461, L41

Martínez-Vázquez C. E. et al., 2016b, MNRAS, 462, 4349

Martínez-Vázquez C. E. et al., 2017, ApJ, 850, 137

McConnachie A. W., 2012, AJ, 144, 4

Menzies J. W., Feast M. W., Whitelock P. A., Matsunaga N., 2011, MNRAS, 414,3492
Monson A. J., Freedman W. L., Madore B. F., Persson S. E., Scowcroft V., Seibert M., Rigby J. R., 2012, ApJ, 759, 146

Monson A. J. et al., 2017, AJ, 153, 96

Muraveva T. et al., 2015, ApJ, 807, 127

Muraveva T. et al., 2018a, MNRAS, 473, 3131

Muraveva T., Garofalo A., Scowcroft V., Clementini G., Freedman W. L., Madore B. F., Monson A. J., 2018b, MNRAS, 480, 4138

Muraveva T., Delgado H. E., Clementini G., Sarro L. M., Garofalo A., 2018c, MNRAS, preprint (arXiv:1805.08742)

Neeley J. R. et al., 2015, ApJ, 808, 11

Neeley J. R. et al., 2017, ApJ, 841, 84

Persson S. E. et al., 2013, PASP, 125, 654

Pietrzyński G. et al., 2008, AJ, 135, 1993

Pietrzyński G. et al., 2013, Nature, 495, 76

Rizzi L., Tully R. B., Makarov D., Makarova L., Dolphin A. E., Sakai S., Shaya E. J., 2007, ApJ, 661, 815

Salaris M., de Boer T., Tolstoy E., Fiorentino G., Cassisi S., 2013, A\&A, 559, A57

Sales L. V., Navarro J. F., Abadi M. G., Steinmetz M., 2007, MNRAS, 379, 1464

Schlafly E. F., Finkbeiner D. P., 2011, ApJ, 737, 103

Sesar B. et al., 2014, ApJ, 793, 135

Shapley H., 1938, Harv. Coll. Obs. Bull., 908, 1

Siegel M. H., 2006, ApJ, 649, L83

Sollima A., Borissova J., Catelan M., Smith H. A., Minniti D., Cacciari C., Ferraro F. R., 2006, ApJ, 640, L43

Stetson P. B., 1987, PASP, 99, 191

Stetson P. B., 1994, PASP, 106, 250

Stierwalt S., Liss S. E., Johnson K. E., Patton D. R., Privon G. C., Besla G., Kallivayalil N., Putman M., 2017, Nature Astron., 1, 0025

Tammann G. A., Sandage A., Reindl B., 2008, ApJ, 679, 52

Tolstoy E. et al., 2004, ApJ, 617, L119

Tolstoy E., Hill V., Tosi M., 2009, ARA\&A, 47, 371

Tully R. B. et al., 2013, AJ, 146, 86

van Agt S. L. T. J., 1978, Publ. David Dunlap Obs., 3, 205

Webbink R. F., 1985, Dyn. Star Clusters, 113, 541

Weisz D. R., Dolphin A. E., Skillman E. D., Holtzman J., Gilbert K. M., Dalcanton J. J., Williams B. F., 2014, ApJ, 789, 147

Zinn R., West M. J., 1984, ApJS, 55, 45

\section{SUPPORTING INFORMATION}

Supplementary data are available at MNRAS online.

Table 2. Photometry of Sculptor RRL at $3.6 \mu \mathrm{m}$.

Fig. A1. Light curves in the [3.6]-band for our sample of RRLs in Sculptor (they are the continuation of Figure 2).

Fig. A2. Same as Fig. A1. Grey filled squares mark data points that were discarded when fitting the light curves with GRATIS. V2021 is a problematic star not used to fit the PL relations (see Section 3.1 and Table 1).

Fig. A3. Same as Fig. A2. Both the period derived from the analysis of our data with GRATIS and the original period from K95 were used to fold the light curve of V3468 in the bottom left two panels (see Section 3.1 for details).

Please note: Oxford University Press is not responsible for the content or functionality of any supporting materials supplied by the authors. Any queries (other than missing material) should be directed to the corresponding author for the article.

This paper has been typeset from a $\mathrm{T}_{\mathrm{E}} \mathrm{X} / \mathrm{LAT} \mathrm{T}$ file prepared by the author. 